\title{
Regenerative multibunch beam breakup instabilities and countermeasures for a high-intensity electron accelerator and the superconducting Darmstadt linear electron accelerator
}

\author{
Sergei Glukhov®, ${ }^{1,2, *}$ Oliver Boine-Frankenheim, ${ }^{1,3}$ Michaela Arnold $\odot,{ }^{4}$ and Christian Stoll ${ }^{5}$ \\ ${ }^{1}$ Technische Universität Darmstadt, Schlossgartenstrasse 8, 64289 Darmstadt, Germany \\ ${ }^{2}$ Budker Institute of Nuclear Physics SB RAS, Novosibirsk 630090, Russia \\ ${ }^{3}$ GSI Helmholtzzentrum für Schwerionenforschung GmbH, Planckstrasse 1, 64291 Darmstadt, Germany \\ ${ }^{4}$ Institut für Kernphysik, Technische Universität Darmstadt, \\ Schlossgartenstr. 9, D-64289 Darmstadt, Germany \\ ${ }^{5}$ Institut für Kernphysik, Johannes Gutenberg-Universität Mainz, \\ Johann-Joachim-Becher-Weg 45, D-55128 Mainz, Germany
}

(Received 21 October 2021; accepted 10 January 2022; published 4 February 2022)

\begin{abstract}
Regenerative multibunch beam breakup instabilities are a well-known phenomenon in recirculating linacs where particle bunches pass multiple times through the same superconducting rf cavities with extremely high quality factor. This is in particular true for energy recovery linacs. Parasitic electromagnetic modes excited in the cavities can affect bunch dynamics in such a way, that on its subsequent passes it excites the modes further and a positive feedback loop is formed. Direct bunch tracking and a stability analysis technique can be used to study the instability. Usually only dipole modes are considered. In the present work, similar approaches are applied to monopole and quadrupole modes and illustrated with simulation results for the S-DALINAC and MESA facilities. An approximated stability analysis technique with better performance for the case of multiple recirculations is proposed. Countermeasures including betatron phase advance adjustment and additional betatron coupling are considered and a universal criterion for assessment of their effectiveness is proposed. A simple model of a damped oscillator with feedback is proposed as a universal example illustrating the phenomenon in general.
\end{abstract}

DOI: $10.1103 /$ PhysRevAccelBeams.25.024402

\section{INTRODUCTION}

Recirculating linac can be regarded as a hybrid of a conventional linac and a storage ring. This concept allows multiple acceleration of the particles using the same accelerating structure, to achieve higher energies as in storage rings. At the same time the high beam quality is preserved as in linacs. Moreover, it allows recovering the energy from the beam by passing it through the accelerating structure in decelerating phase before ejection. This technique is implemented in so called energy recovery linacs (ERLs) [1].

However, the concept of a recirculating linac brings not only advantages but also a new type of instability, which was not possible in conventional linacs: regenerative multibunch beam breakup (BBU) instability. It is caused by parasitic electromagnetic modes, that are excited in the

\footnotetext{
*sergei.glukhov@tu-darmstadt.de
}

Published by the American Physical Society under the terms of the Creative Commons Attribution 4.0 International license. Further distribution of this work must maintain attribution to the author(s) and the published article's title, journal citation, and DOI. accelerating structure. Their negative effect was already known and well-studied for conventional linacs [2]. In recirculating machines, where the same particles pass several times through the same cavity, formation of a feedback loop is possible. Therefore, the achievable beam current may be limited at levels much lower than that of conventional linacs.

Usually parasitic modes are referred to as higher-order modes (HOMs) because their azimuthal symmetry order is higher than that of the fundamental mode (the mode used for acceleration/deceleration of the beam) which always has monopole symmetry. In the present work they will be referred to simply as modes because there are parasitic monopole modes; moreover, interaction with the fundamental mode can be neglected in BBU study (only energy variation along the beam path should be taken into account). It is the reason for another naming convention: recirculating linacs and ERLs are not distinguished here and both named recirculating machines.

Usually only dipole modes are considered as the source of the instability, but it can be also triggered by monopole and quadrupole modes. It will be shown how the effect of all these modes can be studied in a very similar way and only in terms of linear beam dynamics. 
BBU studies are already an essential design part for new machines but they can be also very useful for existing facilities. Two different techniques are widely used: bunch tracking and stability analysis. The most complete theory exists for dipole modes [3,4,5], also there are several papers on monopole [6] and quadrupole [7] modes. The present study is an attempt to compile all these approaches into a universal approach taking advantage of the existence of two completely different techniques which can be crosschecked.

One of the main questions which can be answered within BBU studies is how to increase the threshold current. It can be done using various means but here the focus is on the simplest ones. Techniques related only to recirculation arc optics manipulation were already proposed and studied in [5]. Here an attempt is taken to consider them in a more general way.

Recently there are several recirculating machines in operation or in design/construction phase, e.g., S-DALINAC (Darmstadt, Germany) [8], MESA (Mainz, Germany) [9], bERLinPro (Berlin, Germany) [10], CBETA (USA) [11], PERLE (Orsay, France) [12], NovoFEL (Novosibirsk, Russia) [13]. Examples in the present work are given for the first two facilities in this list.

\section{MESA AND S-DALINAC FACILITIES}

MESA (Mainz Energy recovery Superconducting Accelerator) is a recirculating machine under construction at Johannes Gutenberg University in Mainz. It will be operated in the External Beam (EB) mode (as a recirculating linac) with $150 \mu \mathrm{A}$ electron beam at $155 \mathrm{MeV}$ and in the Energy Recovery (ER) mode (as an ERL) with $1 \mathrm{~mA}$ (first stage) and later $10 \mathrm{~mA}$ (second stage) electron beam at $105 \mathrm{MeV}$.

S-DALINAC is a recirculating machine which is in operation at Technische Universität Darmstadt since 1991 as a twice recirculating linac [8]. During a major upgrade in 2015/2016 the third recirculation was added as well as ERL operation mode. In thrice-recirculating mode the maximum beam energy is $130 \mathrm{MeV}$ at design beam currents up to $20 \mu \mathrm{A}[14,15]$. In ERL operation beam energies of up to $68 \mathrm{MeV}$ are possible. First operation in ERL mode was achieved in 2017 [16].

Both facilities can operate in CW (Continuous Wave) mode. The present paper describes results of BBU studies only for the ER mode of MESA and for the twicerecirculating ERL mode of S-DALINAC. Schemes of the facilities corresponding to these operation modes with naming of the recirculation arcs are presented in Figs. 1 and 2.

MESA uses four 9-cell $1.3 \mathrm{GHz}$ TESLA-type superconducting rf cavities [17] grouped into two accelerating modules. In the ER mode the beam passes the arcs in the following order: $\mathrm{T} 1 \rightarrow \mathrm{T} 2 \rightarrow \mathrm{T} 3 \rightarrow \mathrm{PIT} \rightarrow \mathrm{T} 3 \rightarrow \mathrm{T} 2 \rightarrow \mathrm{T} 1$. The length of the PIT (Pseudo-Internal Target) arc equals

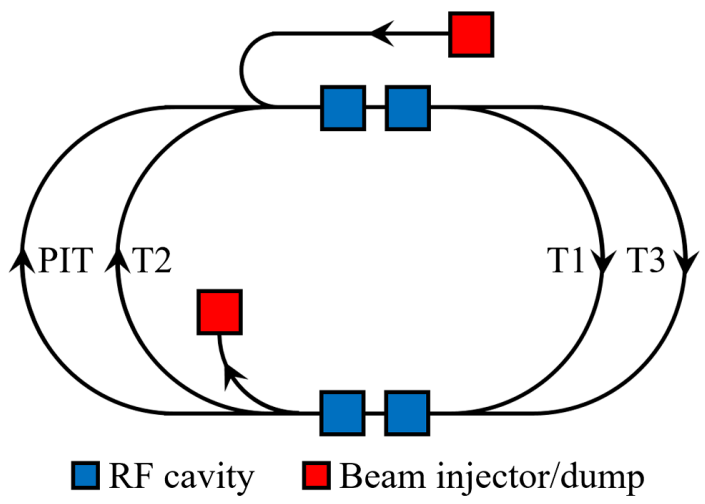

FIG. 1. The MESA facility scheme (ER mode) used in this study.

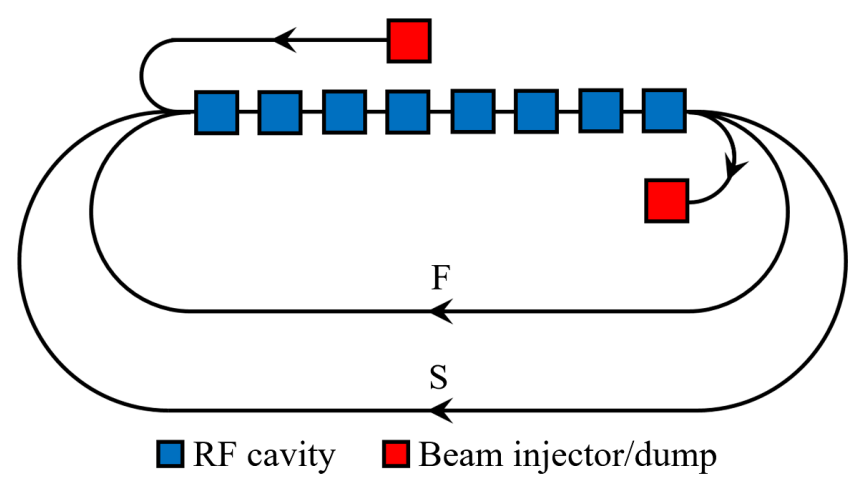

FIG. 2. The S-DALINAC facility scheme (twice recirculating ERL mode) used in this study.

half-integer $\mathrm{rf}$ wavelengths, therefore, particles gain 12.5 MeV on the first two passes through each cavity and lose the same amount on the second two. The injection energy is $5 \mathrm{MeV}$, so the maximal gained energy in this mode is $105 \mathrm{MeV}$.

S-DALINAC uses eight 20-cell $3 \mathrm{GHz}$ superconducting rf cavities [18] (not including the cavities in the injector linac which are not relevant for BBU study). In the twicerecirculating ERL mode the beam passes the arcs in the following order: $F \rightarrow S \rightarrow F$. The length of the $S$ arc is adjusted using dedicated equipment [19] to be equal to halfinteger rf wavelengths, therefore particles gain energy in the cavities before passing this arc and lose energy after. The rf phases and corresponding energy gain/loss are set individually for each cavity to provide the best particle transmission.

\section{BBU SIMULATION MODEL}

For the purpose of BBU simulations a recirculating machine can be represented as a sequence of rf cavities connected by lattice regions in the order seen by one bunch on its way from injector to beam dump or ejection. Each cavity and lattice region may appear several times in this 
sequence depending on the topology of the machine. Set of modes which can be excited in each cavity should be defined.

The beam is represented as a sequence of equidistant bunches injected into the machine. Interval between adjacent bunches is $t_{b}$, average beam current is $I_{0}$. Each bunch is characterized with its total charge $q=I_{0} t_{b}$, centroid vector $\mathbf{X}$ and second-order distribution moment matrix $\boldsymbol{\Sigma}$ in accelerator coordinates:

$$
\mathbf{X}=\left(x, p_{x}, y, p_{y}, z, p_{z}\right)^{T}, \quad \mathbf{\Sigma}=\left\langle\mathbf{X}^{T} \mathbf{X}\right\rangle,
$$

where $\langle\ldots\rangle$ denotes averaging over particles of the bunch. Usage of different injection patterns and higher-order distribution moment tensors is also possible and planned for the future study.

Each cavity is considered as a set of thin rf-multipoles (one for each mode which can be excited) placed at the center of the physical cavity. Each mode $\lambda$ is characterized with azimuthal symmetry order $n_{\lambda}$ (0 for monopole modes, 1 for dipole mode, etc.), frequency $f_{\lambda}$ (or angular frequency $\omega_{\lambda}=2 \pi f_{\lambda}$ ), quality factor $Q_{\lambda}$ (or damping factor $\left.\Gamma_{\lambda}=\frac{\omega_{\lambda}}{2 Q_{\lambda}}\right)$, and $(R / Q)_{\lambda}$. Modes with $n_{\lambda} \geq 1$ also have polarization angle $\theta_{\lambda}$ and always appear in pairs (for axially symmetric cavity the only difference between mode properties within a pair is $\left.2\left|\theta_{\lambda_{2}}-\theta_{\lambda_{1}}\right|=\pi / n_{\lambda}\right)$.

An important remark is needed concerning $(R / Q)_{\lambda}$. This quantity shows the efficiency of energy transfer between the electromagnetic field in the cavity and the beam passing through it. It is defined as follows [20]:

$$
\left(\frac{R}{Q}\right)_{\lambda}=\frac{\left|V_{\mathrm{acc}}\right|^{2}}{2 \omega_{\lambda} W}
$$

where $V_{\text {acc }}$ is the accelerating voltage seen by the particle passing through the cavity (in complex representation), $W$ is the total energy stored in the cavity. This definition works only for monopole modes, therefore, in the present paper a slightly modified quantity $(R / Q)_{\lambda}^{\prime}$ will be used instead:

$$
\left(\frac{R}{Q}\right)_{\lambda}^{\prime}=\frac{\left.\left|V_{\mathrm{acc}}\right|_{r=r_{0}, \varphi=\theta_{\lambda}}\right|^{2}}{2 \omega_{\lambda} W r_{0}{ }^{2 n_{\lambda}}},
$$

where $r_{0}$ is a small shift of the particle's trajectory from the cavity axis. Note that $(R / Q)_{\lambda}^{\prime}$ has different units for different $n_{\lambda}$.

Field patterns for a variety of eigenmodes in MESA and S-DALINAC cavities have been calculated using CST MICROWAVE STUDIO [21]. For each mode with significant values of $(R / Q)_{\lambda}$ near the axis, this quantity depends on transversal coordinates in a similar way as for a thin rf-multipole containing a mode $\mathrm{TM}_{n_{\lambda} 10}$. Therefore, in simulations each mode was represented with the corresponding $\mathrm{TM}_{n_{\lambda} 10}$ rf-multipole with the same $\omega_{\lambda}, \Gamma_{\lambda}$ and $\theta_{\lambda}$ values. It is worth noting that for implementation of such a replacement in a real machine not necessarily thin pillbox cavities would be used because there is an unambiguous relationship between $\omega_{\lambda}$ and $(R / Q)_{\lambda}$ for modes in a pillbox cavity. "Pillbox-like" cavities with unchanged fields near the axis but modified outer contour would be used instead to obtain different expression for stored energy $W$ in Eq. (1) providing the required $(R / Q)_{\lambda}$ value. Exact shape is not important here since only fields near the cavity axis are of interest. Electric field has only longitudinal component and may be approximated as follows:

$E_{z}(r, \varphi, t)=\frac{E_{0}}{n_{\lambda} !}\left(\frac{\omega_{\lambda}}{2 c} r\right)^{n_{\lambda}} \cos \left[n_{\lambda}\left(\varphi-\theta_{\lambda}\right)\right] e^{\left(i \omega_{\lambda}-\Gamma_{\lambda}\right) t}$,

where $E_{0}$ is a field amplitude; for a thin cavity it can be expressed via complex mode voltage:

$$
E_{0}=\frac{\operatorname{Re} V_{\lambda}}{\Delta L},
$$

where $\Delta L \rightarrow 0$ is the cavity length. Usability of the definition Eq. (2) here becomes obvious because in this approximation $(R / Q)_{\lambda}^{\prime}$ is independent on $r_{0}$ and completely characterizes energy transfer between a bunch and a mode with arbitrary $n_{\lambda}$.

Therefore, voltage gain of the mode after passage of a short bunch with transversal distribution density $\rho(x, y)$ can be written as follows:

$$
\Delta V_{\lambda}(t)=\frac{\omega_{\lambda}}{2}\left(\frac{2 c}{\omega_{\lambda}}\right)^{n_{\lambda}}\left(\frac{R}{Q}\right)_{\lambda}^{\prime} \hat{M}_{\lambda}
$$

where

$$
\hat{M}_{\lambda}=\int \rho(r, \varphi) r^{n_{\lambda}+1} \cos \left[n_{\lambda}\left(\varphi-\theta_{\lambda}\right)\right] \mathrm{d} r \mathrm{~d} \varphi .
$$

The integral used here has clear properties of a beam distribution moment but also depends on the mode polarization angle $\theta_{\lambda}$. Therefore, $\hat{M}_{\lambda}$ can be called bunch-mode interaction moment or simply interaction moment. Also specific interaction moment can be defined as $\hat{M}_{\lambda}$ per unit bunch charge:

$$
\hat{m}_{\lambda}=\frac{\hat{M}_{\lambda}}{\int \rho(r, \varphi) r \mathrm{~d} r \mathrm{~d} \varphi} .
$$

It will be used in the next section devoted to stability analysis.

The described energy transfer scheme is only one half of the mechanism of the feedback loop formation which possibly leads to the BBU instability. The second half is provided via slightly different schemes for different $n_{\lambda}$. For monopole modes $\left(n_{\lambda}=0\right)$ this is a relation between average bunch momentum and arrival time to a cavity 
via $T_{56}$ transport matrix element. Bunches interacted with a parasitic monopole mode change their average momentum by $\Delta p$ (in addition to the interaction with the fundamental mode) and arrive next time to the same cavity sooner or later $\left(\Delta t=\frac{T_{56}}{c} \frac{\Delta p}{p}\right)$. If this change leads to a more effective energy transfer from the bunch to the cavity and the loss rate is not high enough to dissipate this energy from the parasitic mode, then instability arises.

Interaction of on-axis bunches with a $n_{\lambda} \geq 1$ mode does not change their average momentum but they are affected by magnetic field of the mode (which is negligible near the axis for $n_{\lambda}=0$ ). Using Eq. (3) and Maxwell's equations one can obtain an expression for magnetic field near the cavity axis. This is the field of a magnetic multipole, the same as those used for representation of thin dipole, quadrupole, etc., lenses in lattice design software such as elegant [22] and MAD-X [23]. Such elements are characterized with multipole order (corresponds to $n_{\lambda}$ ), tilt angle (rotation around beam axis, corresponds to $\theta_{\lambda}$ ) and $2 n_{\lambda}$-pole focusing strength:

$$
K_{2 n_{\lambda}}=\frac{G_{n_{\lambda}-1}}{B \rho},
$$

where $B \rho$ is the magnetic rigidity defined by charge $q_{e}$ of a single particle in a beam and its momentum $p_{\lambda}$ at the time instant of passing the element:

$$
B \rho=\frac{p_{\lambda}}{q_{e}},
$$

$G_{n_{\lambda}-1}$ is a $2 n_{\lambda}$-pole gradient integrated over the element's length. In case of zero tilt angle $\left(\theta_{\lambda}=0\right)$ it can be expressed as follows:

$$
G_{n_{\lambda}-1}=\int \frac{\partial^{n_{\lambda}-1} B_{y}}{\partial x^{n_{\lambda}-1}} \mathrm{~d} L
$$

Using Eqs. (3), (4) and Maxwell's equations one can rewrite focusing strength, the expression is independent on $\theta_{\lambda}$ :

$$
K_{2 n_{\lambda}}=\frac{q_{e}}{p_{\lambda} \omega_{\lambda}}\left(\frac{\omega_{\lambda}}{2 c}\right)^{n_{\lambda}} \operatorname{Im} V_{\lambda}
$$

Therefore, complex mode voltages $V_{\lambda}$ (measured in Volts for any $n_{\lambda}$ ) are the most convenient quantities for describing current state of the modes in simulations.

Lattice regions between centers of two cavities (or two passages of the same cavity) are characterized with $6 \times 6$ transport matrix $\mathbf{T}$, reference momentum $p$, corresponding particle velocity $v$ and time-of-flight $\Delta t$. For brevity, these regions will be further referred to as arcs. Particle momentum value $p_{\lambda}$ at the time instant of passing the cavity containing mode $\lambda$ in simulations is chosen to be an average of reference momenta of two adjacent arcs.

A Python script for BBU simulations has been developed. It is organized in the following way. Sequence of $N_{a}$ arcs and pointlike cavities between them seen by a bunch is unrolled onto the positive longitudinal semiaxis $z$. $N_{b}$ bunches are placed initially onto the negative $z$-semiaxis and given with a velocity corresponding to the injection energy. Bunch spacing is chosen according to the required injection rate. During the simulations bunch sequence is moved forward along $z$-axis until the last bunch reaches the beam dump. Bunch velocity depends on arc's reference momentum. Calculations should be performed only at instants when a bunch encounters a cavity, they consist of three tasks: 1. Bunch coordinate transformation through the previous arc:

$$
z \mapsto z+v \Delta t_{b}, \quad \mathbf{X} \mapsto \mathbf{T X}, \quad \mathbf{\Sigma} \mapsto \mathbf{T} \boldsymbol{\Sigma} \mathbf{T}^{T},
$$

where $\Delta t_{b}$ is the time passed since the previous calculation done for this bunch. 2. Voltage recalculation for all modes excited in this cavity:

$$
V_{\lambda} \mapsto V_{\lambda} e^{\left(i \omega_{\lambda}-\Gamma_{\lambda}\right)\left(\Delta t_{c}+z / v\right)},
$$

where $\Delta t_{c}$ is the time passed since the previous recalculation done for this cavity. 3. Simulation of all bunchmode interactions (depends on $n_{\lambda}$ ).

For monopole modes $\left(n_{\lambda}=0\right)$ :

$$
\begin{aligned}
X_{6} \mapsto X_{6}+\frac{q_{e}}{p_{\lambda} c} \operatorname{Re}\left(V_{\lambda}-\frac{1}{4} q \omega_{\lambda}\left(\frac{R}{Q}\right)_{\lambda}^{\prime}\right), \\
V_{\lambda} \mapsto V_{\lambda}-\frac{1}{2} q \omega_{\lambda}\left(\frac{R}{Q}\right)_{\lambda}^{\prime} .
\end{aligned}
$$

For dipole modes $\left(n_{\lambda}=1\right)$ :

$$
\begin{aligned}
V_{\lambda} & \mapsto V_{\lambda}+q c\left(\frac{R}{Q}\right)_{\lambda}^{\prime}\left(X_{1} \cos \theta_{\lambda}+X_{3} \sin \theta_{\lambda}\right), \\
\mathbf{X} & \mapsto \mathbf{X}+\frac{q_{e} \operatorname{Im} V_{\lambda}}{2 p_{\lambda} c}\left(0, \cos \theta_{\lambda}, 0, \sin \theta_{\lambda}, 0,0\right)^{T} .
\end{aligned}
$$

For quadrupole modes $\left(n_{\lambda}=2\right)$ :

$$
\begin{aligned}
& V_{\lambda} \mapsto V_{\lambda}+\frac{2 q c^{2}}{\omega_{\lambda}}\left(\frac{R}{Q}\right)_{\lambda}^{\prime}\left(\left(\Sigma_{11}-\Sigma_{33}\right) \cos 2 \theta_{\lambda}+2 \Sigma_{13} \sin 2 \theta_{\lambda}\right), \\
& \mathbf{X} \mapsto \mathbf{T}_{q} \mathbf{X}, \quad \boldsymbol{\Sigma} \mapsto \mathbf{T}_{q} \boldsymbol{\Sigma} \mathbf{T}_{q}^{T},
\end{aligned}
$$

where 


$$
\begin{aligned}
\mathbf{T}_{q} & =\left(\begin{array}{cccccc}
1 & 0 & 0 & 0 & 0 & 0 \\
-P \cos 2 \theta_{\lambda} & 1 & P \sin 2 \theta_{\lambda} & 0 & 0 & 0 \\
0 & 0 & 1 & 0 & 0 & 0 \\
P \sin 2 \theta_{\lambda} & 0 & P \cos 2 \theta_{\lambda} & 1 & 0 & 0 \\
0 & 0 & 0 & 0 & 1 & 0 \\
0 & 0 & 0 & 0 & 0 & 1
\end{array}\right), \\
P & =\frac{\omega_{\lambda} q_{e} \operatorname{Im} V_{\lambda}}{4 p_{\lambda} c^{2}} .
\end{aligned}
$$

Bunch order is preserved during the simulations, therefore at each step only the first bunch on each arc should be checked to find the next bunch to encounter a cavity. Thus only $O\left(N_{a}\right)$ operations are needed to simulate each of $N_{a} \times N_{b}$ bunch-mode interactions. Therefore, calculation time grows linearly with $N_{b}$.

To make a decision concerning stability at a given beam current one may approximate all $\left|V_{\lambda}\right|(t)$ dependencies with exponential functions: if there is at least one rising exponent then the current is unstable. Then, e.g., dichotomy method can be used to find the threshold current value with required accuracy, as in bi code by I. Bazarov [24]. However the number of injected bunches $N_{b}$ should be large enough to mitigate the influence of the transition process in the beginning of the tracking. Also this technique is not applicable in case of nonzero equilibrium voltages. This is a natural situation for quadrupole modes because bunches cannot be round or pointlike in all cavities. It is also possible for dipole modes when reference orbit is off-axis in cavities, e.g., due to misalignments. In such cases the following alternative criterion can be used: time period $N_{b} t_{b}$ between entrance of the first and the last bunch into the lattice is divided in 4 equal parts, the first two quarters are discarded to exclude transition process, then $\left|V_{\lambda}\right|$ dispersion values in the third and fourth quarters are compared. If $\left(\mathrm{D}\left|V_{\lambda}\right|\right)_{3}>$ $\left(\mathrm{D}\left|V_{\lambda}\right|\right)_{4}$ then the current is stable and vice versa.

\section{STABILITY ANALYSIS}

\section{A. Mode-mode interaction}

After development of the BBU simulation algorithm and determining main instability drivers, one may want to find a way how to decide whether system is stable or not without direct simulation. It is worth noting that at the level where BBU-related phenomena become important, the effect of every single bunch-mode interaction is small. This is also the case for the net effect of all such interactions on a single bunch during its passage through the lattice. Freshly injected bunches have no BBU-related information and receive it only from the modes, where it was deposited by preceding bunches. Moreover, lifetime of a mode is much larger than that of a single bunch and even potentially infinite for $\mathrm{CW}$ operation. Therefore, it makes sense to consider modes as main actors and bunches as an interaction medium. Then one needs to switch from bunch-mode interactions to mode-mode interactions, which should be studied separately for different $n_{\lambda}$.

Using coordinate and mode voltage transformations mentioned in the previous section, one can express all mode voltages via their values at previous instants of time. Stability analysis technique for dipole modes developed in $[3,4,5]$ makes use of the fact that for $n_{\lambda}=1$ under the assumptions made so far resulting equations are linear. Additional linearization was applied in [6] to use similar technique for monopole modes and only a special case where the equations are linear for quadrupole modes was considered in [7]. The present paper makes an attempt to summarize all these cases. As in the papers mentioned above, all equations are linearized by mode voltages where needed, therefore, superposition principle holds for them. This means that all interactions between modes are pairwise and independent (including interaction of a mode with itself). Moreover, interactions between the same modes but via different number of recirculations are also independent.

\section{B. Basic definitions}

In the previous section the bunch-mode interaction moment $\hat{M}_{\lambda}$ and the specific bunch-mode interaction moment $\hat{m}_{\lambda}$ were defined. Now one can define complex wake potential as a complex mode voltage per unit interaction moment:

$$
W_{\lambda}(t)=\frac{\mathrm{d} V_{\lambda}(t)}{\mathrm{d} \hat{M}_{\lambda}}
$$

More convenient quantities (used, e.g., in [3]) are longitudinal $W_{l \lambda}$ and transversal $W_{t \lambda}$ wake potentials which are simply real and imaginary parts of $W_{\lambda}$ :

$$
W_{l \lambda}=\operatorname{Re} W_{\lambda}, \quad W_{t \lambda}=\operatorname{Im} W_{\lambda} .
$$

Then complex mode voltage can be expressed as follows:

$$
V_{\lambda}(t)=\int_{-\infty}^{t} W_{\lambda}\left(t-t^{\prime}\right) I\left(t^{\prime}\right) \hat{m}_{\lambda}\left(t^{\prime}\right) \mathrm{d} t^{\prime}
$$

where $I(t)$ is the beam current. For bunched beam in case of pointlike bunches, average current $I_{0}$ and bunch spacing $t_{b}$ it can be expressed as follows:

$$
I(t)=\sum_{m=-\infty}^{\infty} I_{0} t_{b} \delta_{D}\left(t-m t_{b}\right),
$$

where $\delta_{D}$ is the Dirac delta function.

After substitution of Eq. (8) into Eq. (7) the integral in Eq. (7) is readily calculated and transformed into infinite sum. To proceed analytically, one needs it to be a sum of a geometric series. This is possible when for all $V_{\lambda}$ the following condition is fulfilled: 


$$
V_{\lambda}\left(t+t_{b}\right)-\tilde{V}_{0 \lambda}=\left(V_{\lambda}(t)-\tilde{V}_{0 \lambda}\right) e^{i \omega t_{b}},
$$

where $\tilde{V}_{0 \lambda}$ is a constant (equilibrium value in the case of stable motion). Thus, $V_{\lambda}$ can be expressed as a series:

$$
V_{\lambda}(t)=\tilde{V}_{0 \lambda}+\sum_{n=-\infty}^{\infty} V_{n \lambda} e^{i\left(\omega+\frac{2 \pi n}{t_{b}}\right) t}
$$

\section{Multiple recirculations}

If a bunch in a recirculating machine passes the same cavity more than twice, then there are modes interacting with each other via several different paths (which can be also the case for a mode interacting with itself). The simplest way to find all these paths is to unroll the cavities and the arcs between them onto a straight line in the order seen by a bunch and then find all possible transitions from the first to the second mode. Figure 3 illustrates this approach for ER operating mode of MESA (for simplicity each of the two accelerating modules is represented as a single cavity with a single mode). Here $L_{\mu \lambda}$ is the number of different paths between modes $\mu$ and $\lambda$. The approach is also correct for the case of different mode sets excited in the cavities.

Mode should have large Q-factor to cause BBU, thus mode damping during time interval $t_{b}$ is negligible. Moreover, parasitic modes are not in resonance with the beam, i.e., they interact with bunches at nonfixed phases. Therefore, relative phasing of the bunches on different paths between the same modes may be neglected. This approximation leads to simpler formulas and better performance than those of the exact stability analysis technique [4], its correctness can be controlled using cross-check with tracking results.

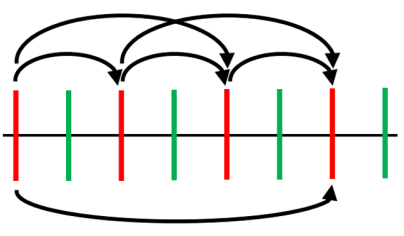

Module $1 \rightarrow$ Module 1, $L_{11}=6$

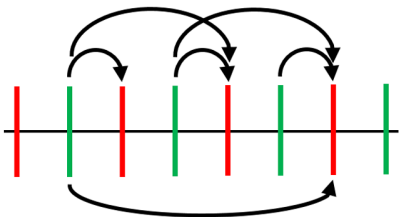

Module $2 \rightarrow$ Module $1, \quad L_{21}=6$

- Module 1

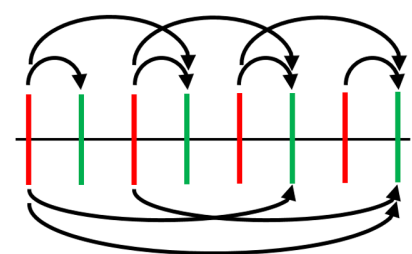

Module $1 \rightarrow$ Module 2, $L_{12}=10$

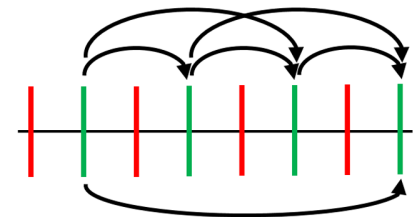

Module $2 \rightarrow$ Module 2, $L_{22}=6$ - Module 2

FIG. 3. Interaction loops between accelerating modules in ER operating mode of MESA.

\section{Dipole and quadrupole modes}

Consider a single bunch of charge $q$, momentum $p_{\mu}$ and second-order moments matrix $\boldsymbol{\Sigma}_{\mu \lambda}^{l}$ interacting with a mode $\mu$, then passing certain region of the lattice with transport matrix $\mathbf{T}_{\mu \lambda}^{l}$ in a time period $t_{\mu \lambda}^{l}$ and then interacting with a mode $\lambda$ (on the $l$ th loop). Its interaction moment with the second mode will depend on the voltage of the first mode (except for monopole modes where specific interaction moment always equals to unity). Effect of all preceding bunch-mode interaction is simply summed up because only linear beam dynamics is considered. Therefore, in case of dipole modes:

$$
\left.\hat{m}_{\lambda}\right|_{n_{\lambda}=1}=\sum_{\mu} \sum_{l=1}^{L_{\mu \lambda}}{ }^{1 l} C_{\mu \lambda} \delta_{\mu}^{\perp}\left(t-t_{\mu \lambda}^{l}\right),
$$

where

$$
\delta_{\mu}^{\perp}(t)=\frac{q_{e}}{p_{\mu} c} \operatorname{Im} V_{\mu}(t)
$$

and the expression for $\stackrel{1}{C}_{\mu \lambda}^{l}$ can be derived from the coordinate transformation Eq. (5):

$$
\begin{aligned}
\stackrel{1}{C}_{\mu \lambda}^{l}= & \left(T_{\mu \lambda}^{l}\right)_{12} \cos \theta_{\lambda} \cos \theta_{\mu}+\left(T_{\mu \lambda}^{l}\right)_{34} \sin \theta_{\lambda} \sin \theta_{\mu} \\
& +\left(T_{\mu \lambda}^{l}\right)_{32} \sin \theta_{\lambda} \cos \theta_{\mu}+\left(T_{\mu \lambda}^{l}\right)_{14} \cos \theta_{\lambda} \sin \theta_{\mu} .
\end{aligned}
$$

Similar expression can be written for quadrupole modes:

$$
\left.\hat{m}_{\lambda}\right|_{n_{\lambda}=2}=\sum_{\mu} \sum_{l=1}^{L_{\mu \lambda}}\left[\stackrel{2}{C}_{\mu \lambda}^{l} \delta_{\mu}^{\perp}\left(t-t_{\mu \lambda}^{l}\right)+\stackrel{2}{C}_{\mu \lambda}^{l}\left(\delta_{\mu}^{\perp}\left(t-t_{\mu \lambda}^{l}\right)\right)^{2}\right],
$$

where term independent on mode voltages is omitted and

$$
\begin{aligned}
\stackrel{2}{C}_{\mu \lambda}^{l}= & a_{\mu \lambda}^{l} \cos \theta_{\mu} \cos \theta_{\lambda}+b_{\mu \lambda}^{l} \sin \theta_{\mu} \cos \theta_{\lambda} \\
& +c_{\mu \lambda}^{l} \cos \theta_{\mu} \sin \theta_{\lambda}+d_{\mu \lambda}^{l} \sin \theta_{\mu} \sin \theta_{\lambda},
\end{aligned}
$$

coefficients $a_{\mu \lambda}^{l}, b_{\mu \lambda}^{l}, \quad c_{\mu \lambda}^{l}$, and $d_{\mu \lambda}^{l}$ are given in the Appendix A. They can be obtained from the coordinate transformation Eq. (6) and have much more complicated form than for dipole modes because of double matrix product in the $\Sigma$-matrix transformation. Coefficient $\underline{C}_{\mu \lambda}$ is zeroed out in case of no betatron coupling, initially round bunches and a special optics between cavities:

$$
\left(\Sigma_{\mu \lambda}^{l}\right)_{11}=\left(\Sigma_{\mu \lambda}^{l}\right)_{33}, \quad\left|\left(T_{\mu \lambda}^{l}\right)_{12}\right|=\left|\left(T_{\mu \lambda}^{l}\right)_{34}\right| .
$$

The latter condition here is more relaxed than that used in [7] (round bunches on both passes). These conditions are not necessarily exactly fulfilled but usually they are a 
guiding line in the machine design because they allow minimization of the quadrupole mode excitation in the cavities. Therefore, for realistic recirculating machines $\underline{2}_{\mu \lambda}^{2 l}$ may be neglected and the same formulas can be used further for dipole and quadrupole modes.

According to [4], integer $\left(n_{\mu \lambda}^{l}\right)$ and fractional $\left(\delta_{\mu \lambda}^{l}\right)$ part of the ratio $t_{\mu \lambda}^{l} / t_{b}$ can be introduced as follows:

$$
t_{\mu \lambda}^{l}=\left(n_{\mu \lambda}^{l}-\delta_{\mu \lambda}^{l}\right) t_{b}, \quad 0 \leq \delta_{\mu \lambda}^{l}<1 .
$$

Substituting $I(t)$ and $\hat{m}(t)$ into Eq. (7) one obtains a relation between complex voltage $V_{\lambda}(t)$ and the set of instant values of $\operatorname{Im} V_{\lambda}(t)$. Taking imaginary part of its both sides transforms $W_{\lambda}(t)$ into $W_{t \lambda}(t)$. For the purpose of stability analysis only one harmonic from Eq. (9) may be used:

$$
V_{\lambda}(t)=V_{0 \lambda} e^{i \omega t}
$$

Then at $t=0$ one obtains the relation between $\operatorname{Im} V_{0 \lambda}$ values where Im sign can be omitted:

$$
\begin{aligned}
V_{0 \lambda}= & I_{0} \sum_{\mu} V_{0 \mu} \sum_{l=1}^{L_{\mu \lambda}} \frac{n_{\lambda}}{K_{\mu \lambda}^{l}} \frac{n_{\mu \lambda}^{l}}{2 i} \sum_{m=-\infty} e^{\Gamma_{\lambda} m t_{b}} \\
& \times\left(e^{-i \omega_{\lambda} m t_{b}}-e^{i \omega_{\lambda} m t_{b}}\right) e^{-i \omega m t_{b}} e^{i \omega t_{\mu \lambda}}
\end{aligned}
$$

where

$$
\stackrel{n}{K}_{\mu \lambda}^{l}=\frac{\stackrel{n}{C}_{\mu \lambda}^{l} q_{e}}{2 p_{\mu}}\left(\frac{R}{Q}\right)_{\lambda}^{\prime} t_{b}, \quad n_{\lambda}=1,2 .
$$

It can be rewritten in a matrix form:

$$
\frac{1}{I_{0}} \mathbf{V}_{0}=\mathbf{W}(\omega) \mathbf{V}_{0}
$$

where the elements of the matrix $\mathbf{W}(\omega)$ after summation of geometric series become:

$$
\begin{aligned}
W_{\mu \lambda}(\omega)= & \sum_{l=1}^{L_{\mu \lambda}} \frac{n_{\lambda}}{K_{\mu \lambda}^{l}} \frac{e^{i \omega n_{\mu \lambda}^{l} t_{b}-\delta_{\mu \lambda}^{l} \Gamma_{\lambda} t_{b}}}{2 i} e^{-i \delta_{\mu \lambda}^{l} \omega_{\lambda} t_{b}} \\
& \times\left(\frac{e^{-\omega_{\lambda} \omega_{\lambda} t_{b}}}{1-e^{\left(-\Gamma_{\lambda}+i \omega+i \omega_{\lambda}\right) t_{b}}}-\frac{e^{\left(-\Gamma_{\lambda}+i \omega-i \omega_{\lambda}\right) t_{b}}}{1-} .\right.
\end{aligned}
$$

Therefore, the $1 / I_{0}$ values are simply eigenvalues of the $\mathbf{W}(\omega)$ matrix. The result corresponds to that obtained in [4] but the approach used here allows one to apply it also to quadrupole modes.

In the exact theory proposed in [4] the sizes of the $\mathbf{W}(\omega)$ matrix equal to the number of active modes excited in all cavities multiplied by the number of recirculations. Here it is just the number of active modes, which leads to better performance for multiple recirculations case.

\section{E. Monopole modes}

Equation (7) is also correct for monopole modes. $\hat{M}_{\lambda} \equiv 1$ for $n_{\lambda}=0$, however, its right-hand side depends on mode voltages anyway because expression for beam current in Eq. (8) should be rewritten:

$$
I_{\lambda}(t)=\sum_{m=-\infty}^{\infty} I_{0} t_{b} \delta_{D}\left(t-m t_{b}-\Delta t_{m \lambda}\right),
$$

where

$$
\Delta t_{m \lambda}=\sum_{\mu} \sum_{l=1}^{L_{\mu \lambda}} \frac{\left(T_{\mu \lambda}^{l}\right)_{56} q_{e}}{p_{\mu} c^{2}} \operatorname{Re} V_{\mu}\left(m t_{b}-t_{\mu \lambda}^{l}\right) .
$$

In all real cases longitudinal motion does not change the order of the bunches, therefore, this expression can be interpreted as a beam current modulation by mode voltages. If this modulation is weak enough that

$$
\omega_{\lambda} \Delta t_{m \lambda} \ll 1, \quad \forall m, \lambda,
$$

then Eq. (7) can be linearized to matrix form Eq. (11) with

$$
\begin{aligned}
W_{\mu \lambda}(\omega)= & \sum_{l=1}^{L_{\mu \lambda}} \frac{0^{l}}{2} \\
& \times\left\{\omega_{-}\left[\frac{e^{i\left(\omega t_{\mu \lambda}^{l}+\omega_{\mathrm{mm}} \delta_{\mu \lambda}^{l} t_{b}\right)}}{1-e^{i \omega_{\mathrm{mm}} t_{b}}}+\frac{e^{-i\left(\omega t_{\mu \lambda}^{l}+\omega_{\mathrm{pm}} \delta_{\mu \lambda}^{l} t_{b}\right)}}{1-e^{-i \omega_{\mathrm{pm}} t_{b}}}\right]\right. \\
& \left.-\omega_{+}\left[\frac{e^{i\left(\omega t_{\mu \lambda}^{l}+\omega_{\mathrm{mp}} \delta_{\mu t}^{l} t_{b}\right)}}{1-e^{i \omega_{\mathrm{mp}} t_{b}}}+\frac{e^{-i\left(\omega t_{\mu \lambda}^{l}+\omega_{\mathrm{pp}} \delta_{\mu \lambda}^{l} t_{b}\right)}}{1-e^{-i \omega_{\mathrm{pp}} t_{b}}}\right]\right\},
\end{aligned}
$$

where

$$
\begin{gathered}
\omega_{-}=\omega_{\lambda}-i \Gamma_{\lambda}, \quad \omega_{+}=\omega_{\lambda}+i \Gamma_{\lambda}, \quad \omega_{\mathrm{mm}}=\omega-\omega_{-}, \\
\omega_{\mathrm{mp}}=\omega-\omega_{+}, \quad \omega_{\mathrm{pm}}=\omega+\omega_{-}, \quad \omega_{\mathrm{pp}}=\omega+\omega_{+}, \\
\stackrel{K}{\mu \lambda}_{\mu \lambda}^{l}=-\frac{\left(T_{\mu \lambda}^{l}\right)_{56} \omega_{\lambda} q_{e}}{4 p_{\mu} c^{2}}\left(\frac{R}{Q}\right)_{\lambda}^{\prime} t_{b} .
\end{gathered}
$$

Simulation results show that if parasitic monopole modes are not excited initially then instability threshold is always reached while assumption Eq. (12) is still valid.

\section{F. Complex current plot}

The eigenvalues of matrix $\mathbf{W}(\omega)$ cannot be found analytically, thus the indirect way is used. There are no infinitely growing solutions for $I_{0}=0$, this means that all $\omega$ 's have negative imaginary parts. An instability emerges when $I_{0}$ exceeds a threshold value, this means that one of 
the $\omega$ 's gets a positive imaginary part. Therefore, $\omega$ should be varied along the real axis within some region which spans the frequencies $\omega_{\lambda}$ of all excited modes. In general, the resulting current values will be complex, then the value with zero imaginary part and the smallest positive real part is the threshold current.

This leads to the complex current plot technique when $I_{0}(\omega)$ values are plotted as a curve on the complex plane and then the closest to zero intersection of this curve with the positive real semiaxis is searched for. But in calculations this curve is represented as a set of points with varying step between them. Moreover, the number of curves is equal to the total number of modes excited in all cavities (in case of the exact stability analysis technique it is also multiplied by the number of recirculations). Eigenvalue solver does not guarantee the order of the eigenvalues. This means that their order may be different for adjacent $\omega$ values. Without special countermeasures this would lead to jumps between curves and possible spurious intersections. The following algorithm was used to solve the problem: for each $I_{0}$ on the current step the closest value from the previous step is found (values with smaller $\left|I_{0}\right|$ are considered first), and values from the current step are sorted accordingly. Then the order of the curves is preserved and for sufficiently small step in $\omega$ intersection $I_{*}$ with real axis between adjacent values $I_{1}$ and $I_{2}$ exists only if $\operatorname{Im} I_{1} \operatorname{Im} I_{2} \leq 0$ and can be found as follows:

$$
I_{*}=\left\{\begin{array}{ll}
\min \left(\operatorname{Re} I_{1}, \operatorname{Re} I_{2}\right), & \operatorname{Im} I_{1}=\operatorname{Im} I_{2}=0 \\
\frac{\operatorname{Re} I_{1} \operatorname{Im} I_{2}-\operatorname{Re} I_{2} \operatorname{Im} I_{1}}{\operatorname{Im} I_{2}-\operatorname{Im} I_{1}}, & \text { otherwise }
\end{array} .\right.
$$

An example of a complex current plot is given later in the text in Fig. 7.

\section{MODES IN MESA AND S-DALINAC CAVITIES}

\section{A. Mode types}

As it was already mentioned, for BBU simulations and stability analysis only $n_{\lambda}, f_{\lambda}, Q_{\lambda},(R / Q)_{\lambda}^{\prime}$, and $\theta_{\lambda}$ parameters of modes to be studied are important. For Python scripts developed as a part of the present work these data were obtained using eigenmode solver of CST MICROWAVE STUDIO and compiled into two tables with numerical values, one for 9-cell MESA cavity and one for 20-cell S-DALINAC cavity.

MESA and S-DALINAC cavities are arrays of coupled cells, therefore, their eigenmodes tend to form passbands. Modes within one passband have the same $n_{\lambda}$, thus passbands may be called monopole, dipole, quadrupole, etc. Modes of a pillbox cavity fall into two groups: TE (transversal electric, with no longitudinal electric field) and TM (transversal magnetic, with no longitudinal magnetic field). MESA and S-DALINAC cavities have axial symmetry but profile of a single cell differs from that of a pillbox cavity, thus such a classification is valid only for
TABLE I. Lowest passbands of the MESA cavity.

\begin{tabular}{lcccc}
\hline \hline$n_{\lambda}$, type & $f_{\text {min }}, \mathrm{GHz}$ & $f_{\text {max }}, \mathrm{GHz}$ & $Q_{\text {ext }_{\text {min }}}$ & $Q_{\text {ext }}{ }_{\max }$ \\
\hline $0, \mathrm{TM}$ & 1.276 & 1.3 & $1.35 \times 10^{6}$ & $4.45 \times 10^{7}$ \\
1, Hy & 1.621 & 1.789 & $4.59 \times 10^{3}$ & $6.31 \times 10^{5}$ \\
1, Hy & 1.799 & 1.889 & $6.02 \times 10^{3}$ & $8.01 \times 10^{5}$ \\
2, Hy & 2.288 & 2.319 & $3.15 \times 10^{5}$ & $1.85 \times 10^{7}$ \\
0, TM & 2.379 & 2.454 & $2.81 \times 10^{4}$ & $3.15 \times 10^{5}$ \\
2, Hy & 2.472 & 2.492 & $1.18 \times 10^{6}$ & $4.93 \times 10^{7}$ \\
1, Hy & 2.475 & 2.586 & $1.19 \times 10^{1}$ & $3.72 \times 10^{3}$ \\
0, TE & 2.487 & 2.506 & $1.21 \times 10^{7}$ & $1.12 \times 10^{10}$ \\
0, TM & 2.676 & 2.773 & $2.66 \times 10^{4}$ & $5.40 \times 10^{5}$ \\
\hline \hline
\end{tabular}

monopole modes. Modes with $n_{\lambda} \geq 1$ may combine TEand TM-like field patterns in different cells, therefore they are usually called hybrid modes (Hy). Most important properties of the lowest passbands for both cavity types are presented in Table I and Table II.

Differences between mode types are, in principle, not important for calculation of their properties, but they may help to determine the modes with large quality factors which may potentially cause BBU. Quality factors used in simulations constitute of two contributions: $Q_{\text {loss }}$ caused by residual losses in superconducting cavity walls and external quality factor $Q_{\text {ext }}$ caused by coupling of the cavity with nearby equipment:

$$
Q=\left(\frac{1}{Q_{\mathrm{loss}}}+\frac{1}{Q_{\mathrm{ext}}}\right)^{-1} .
$$

Most of the modes have $Q_{\text {loss }} \sim 10^{9}$ which does not prevent them from causing BBU. More important is $Q_{\text {ext }}$ which is mainly determined by coupling of the cavity to mode couplers and cutoff tubes attached to the end-cells of the cavity. Both cavity types have power input couplers, MESA cavity additionally has HOM couplers especially designed to extract power from parasitic modes. This design difference explains much larger $Q_{\text {ext }}$ values for several passbands in S-DALINAC cavity compared to that of MESA cavity. But also cutoff frequencies of the cutoff tubes are important. They determine which modes are trapped inside the cavity (and most likely have larger quality factors and cause

TABLE II. Lowest passbands of the S-DALINAC cavity.

\begin{tabular}{lcccc}
\hline \hline$n_{\lambda}$, type & $f_{\text {min }}, \mathrm{GHz}$ & $f_{\text {max }}, \mathrm{GHz}$ & $Q_{\mathrm{ext}_{\min }}$ & \multicolumn{1}{c}{$Q_{\mathrm{ext}_{\max }}$} \\
\hline $0, \mathrm{TM}$ & 2.885 & 2.992 & $5.83 \times 10^{11}$ & $1.96 \times 10^{14}$ \\
$1, \mathrm{Hy}$ & 3.389 & 3.983 & $1.28 \times 10^{1}$ & $6.82 \times 10^{6}$ \\
$1, \mathrm{Hy}$ & 4.009 & 4.337 & $2.42 \times 10^{2}$ & $2.96 \times 10^{4}$ \\
$2, \mathrm{Hy}$ & 4.971 & 5.072 & $6.52 \times 10^{10}$ & $9.93 \times 10^{12}$ \\
$0, \mathrm{TM}$ & 5.113 & 5.402 & $7.63 \times 10^{0}$ & $3.06 \times 10^{4}$ \\
$1, \mathrm{Hy}$ & 5.392 & 5.658 & $8.98 \times 10^{3}$ & $3.49 \times 10^{4}$ \\
$0, \mathrm{TE}$ & 5.485 & 5.528 & $2.68 \times 10^{9}$ & $2.27 \times 10^{10}$ \\
$2, \mathrm{Hy}$ & 5.583 & 5.701 & $4.44 \times 10^{6}$ & $3.23 \times 10^{9}$ \\
\hline \hline
\end{tabular}


TABLE III. Cutoff frequencies of the beampipe near cavities $(\mathrm{GHz})$.

\begin{tabular}{lcc}
\hline \hline$n_{\lambda}$, type & MESA & S-DALINAC \\
\hline 0, TM & 2.942 & 4.623 \\
0, TE & 4.688 & 7.366 \\
1, Hy & 2.252 & 3.539 \\
2, Hy & 3.731 & 5.863 \\
\hline \hline
\end{tabular}

BBU) and which can propagate through the beampipe. Propagating modes have TE or TM type and may be excited by the modes of the same $n_{\lambda}$ and type in the cavity (hybrid modes may excite both types). Cutoff frequencies are presented in Table III. In MESA cavity a few lowest passbands of each type are trapped, while in S-DALINAC cavity larger fundamental frequency and larger cutoff pipe diameter push dipole cutoff frequency inside the lowest dipole passband. Therefore, one may expect dipole and quadrupole modes in these cavities to be of different relative importance in terms of BBU. This assumption will be proven later in Sec. VII with the stability analysis results.

\section{B. Mode separation}

Another property of an eigenmode spectrum which is really important for calculations is mode separation within passbands. It was shown in [4] that if modes in the spectrum do not overlap, then BBU threshold current caused by their combination is close to the minimal threshold current caused by each of them separately. Then during stability analysis eigenvalue problem may be solved for smaller matrices which increases performance.

Mode width may be estimated as follows:

$$
\Delta f_{\lambda}=\frac{f_{\lambda}}{2 Q_{\lambda}} .
$$

According to simulations in CST MICROWAVE STUDIO, within each passband with $n_{\lambda} \geq 1$ separation between adjacent mode pairs is always several orders of magnitude larger than within one mode pair; also separation between adjacent monopole modes and adjacent $n_{\lambda} \geq 1$ mode pairs is always at least one order of magnitude larger than the width of the modes.

Two sets of $f_{\lambda}$ and $Q_{\lambda}$ measurements were performed for two lowest dipole passbands in 4 MESA cavities: during cold tests at DESY Hamburg [25] and horizontal tests at Helmholtz Institut Mainz (HIM) [26]. Measured distances between adjacent modes compared to measured mode widths (mean value and standard deviation) are presented in Fig. 4. As expected, the first series falls into two parts: distances between mode pairs and distances within pairs. According to statistical spread, some modes within pairs may overlap but for different pairs overlapping is unlikely. Therefore, in all subsequent simulations only one

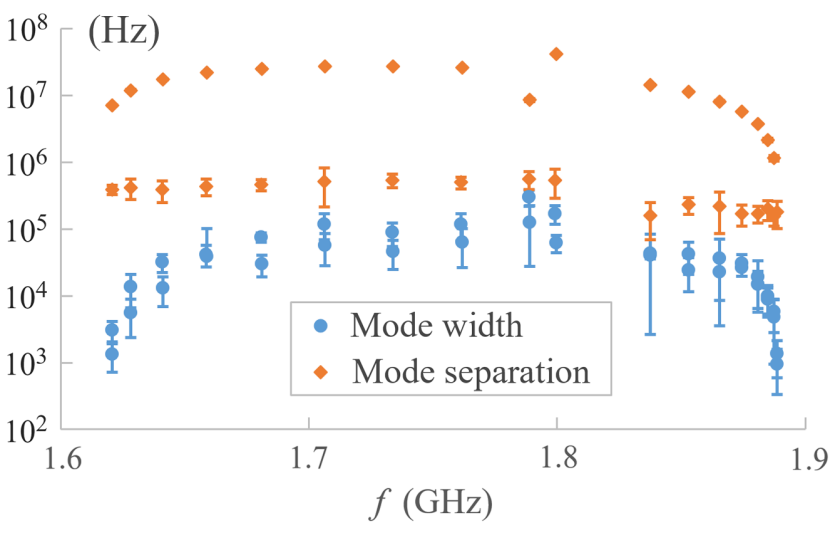

FIG. 4. Mode separation compared to mode width for the lowest two dipole passbands in MESA cavity.

monopole mode or one pair of $n_{\lambda} \geq 1$ modes is excited in each cavity. Successive calculations are performed and minimal threshold current values are taken in case of larger number of active modes.

\section{Parameter randomization}

As it was already seen from the measurements, mode properties may differ from cavity to cavity due to manufacturing tolerances. These small changes in the mode properties are mostly uncontrollable and may lead to significant BBU threshold current variations. Therefore, a correct prediction of the BBU threshold current is not a certain value but a range of values which can be obtained for a set of machine configurations with mode properties generated according to their statistical distributions. Either theoretical [27] or experimental approach can be used for obtaining these distributions. In the present work experimental results from $[25,26]$ were analyzed for this purpose.

Distribution of the measured mode detuning from the simulated frequency values is presented in Fig. 5. It can be approximated with Gaussian distribution with $\sigma=1 \mathrm{MHz}$, nonzero mean value here may be neglected. Distribution of

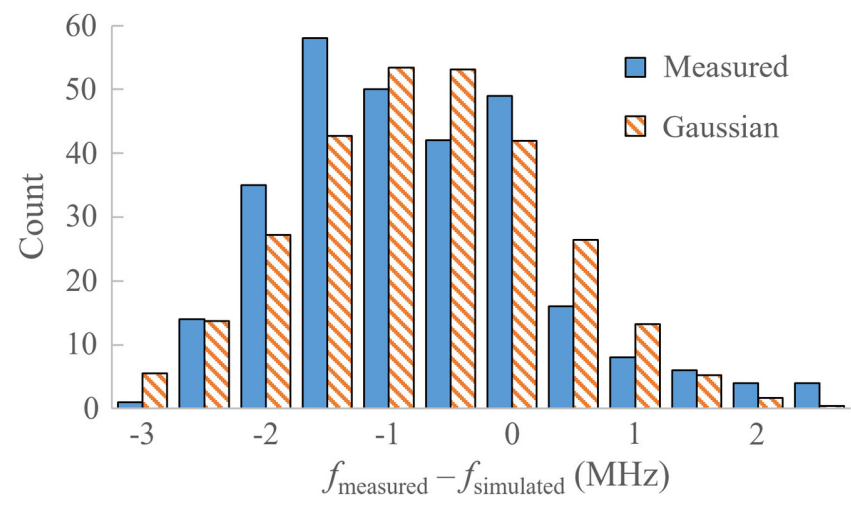

FIG. 5. Measured mode detuning distribution for the lowest two dipole passbands in MESA cavity compared to Gaussian distribution. 


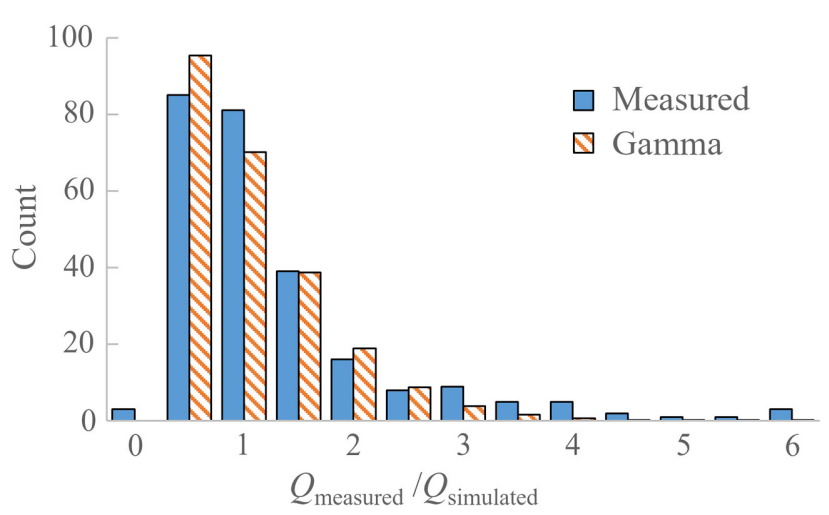

FIG. 6. Measured and simulated quality factor ratio distribution for the lowest two dipole passbands in MESA cavity compared to Gamma-distribution.

the ratio $Q_{\text {measured }} / Q_{\text {simulated }}$ shown in Fig. 6 can be approximated with Gamma-distribution with $\alpha=\beta=2$ providing mean value of 1 . Cutoff at $3 \sigma$ was applied to both distributions to prevent unrealistically large deviations.

Shunt impedance may also differ from cavity to cavity but the effect of its variation on the BBU threshold current is more or less straightforward because $(R / Q)_{\lambda}^{\prime}$ acts only as a common factor. Thus, $(R / Q)_{\lambda}^{\prime}$ was not varied in the present study. The last mode property in the list, polarization angle $\theta_{\lambda}$, is the most affected by manufacturing tolerances, up to full randomization. So, uniform distribution over $\pi / n_{\lambda}$ was used for the $\theta_{\lambda}$ of the first mode in each pair with $n_{\lambda} \geq 1$, the second one was assigned with $\Delta \theta_{\lambda}=\pi /\left(2 n_{\lambda}\right)$.

BBU threshold current depends on the elements of arc transport matrices as well as on mode properties. These matrix elements may also have random errors due to misalignments and power supply errors. This effect was studied in simulations and found to be negligible at the accuracy levels easily achieved in existing machines.

\section{BBU MITIGATION TECHNIQUES}

Different strategies can be used for increasing BBU threshold current in a particular machine. The most obvious way is to decrease quality factors for parasitic modes, which may involve cavity design optimization (especially HOM couplers design optimization) and/or active damping of the modes. Also systems for active damping of the beam oscillations are possible. These strategies imply designing of a dedicated hardware; a separate study is required for each particular case.

Meanwhile, from the formulas mentioned above one can see that there are parameters which are quite easy to change and affect BBU threshold current strongly but in a complicated manner. In linear BBU theory considered here these parameters are transport matrix elements of the $\operatorname{arcs}\left(T_{i j}\right)$ and bunch interval $t_{b}$. Adding of nonlinearities which is planned within the present research will also allow taking chromatic effects into account. Strategy exploiting chromatic decoherence for dipole BBU mitigation was proposed, e.g., in [28].

Effect of bunch interval on BBU threshold current was studied in [5] and found to be negligible. However recently variation of injection pattern was studied and the results are quite promising [29]. A similar study is also planned as a part of the present research.

In this work only transport matrix elements manipulation will be discussed. Optical functions matching for a transport channel is always not a trivial task because horizontal and vertical functions should be matched simultaneously. In case of recirculating machines the task becomes even more complicated because, in fact, they are transport channels with repetitive parts and varying beam energy. In turn, $\mathrm{rf}$ cavities (which are located in these repetitive parts) contribute to beam focusing [30] and this contribution depends on beam energy. Therefore, variation of beam focusing parameters is usually not possible because it most likely leads to intolerable growth of horizontal and/or vertical beam size.

The simplest way to manipulate transport matrix elements without touching optical functions and beam sizes is to use special combinations of beamline elements which do not affect beam distribution but do swap the particles inside it according to a certain law. Such combinations are usually referred to as fictitious beamline elements. Fictitious elements which are to be used for this purpose, would have no effect in absence of BBU because all particles are identical. But each interaction with a mode inside a cavity (which, in fact, causes BBU) gives to every particle a "tag" describing the interaction. Particles get individual sets of these "tags" and become unique, therefore, mixing them one affects beam dynamics.

Only linear beamline elements should be considered within the linear BBU theory, then possible phase space transformations are translations (shifts), rescalings, rotations and reflections. The former two are not appropriate because they do change the beam distribution. Usage of rotations and reflections was studied in [31], several formulas from that work will be cited here. Usage of reflections is restricted by symplecticity condition to the cases of $180^{\circ}$-rotations, therefore, reflections will not be considered in this work.

For brevity in description of possible rotations, one can introduce the following notation for $2 \times 2$ transport matrix block (similar to that used in [5]) of a lattice region with initial optical functions $\beta_{i}, \alpha_{i}$, final optical functions $\beta_{j}, \alpha_{j}$ and betatron phase advance $\phi$ :

$\mathbf{M}_{i j}(\phi)=\left(\begin{array}{cc}\sqrt{\frac{\beta_{j}}{\beta_{i}}}\left(\cos \phi+\alpha_{i} \sin \phi\right) & \sqrt{\beta_{j} \beta_{i}} \sin \phi \\ \frac{\left(\alpha_{i}-\alpha_{j}\right) \cos \phi-\left(1+\alpha_{i} \alpha_{j}\right) \sin \phi}{\sqrt{\beta_{j} \beta_{i}}} & \sqrt{\frac{\beta_{i}}{\beta_{j}}}\left(\cos \phi-\alpha_{j} \sin \phi\right)\end{array}\right)$

Two independent rotations can be performed in $x$ - $p_{x}$ and $y-p_{y}$ planes using a fictitious element phase trombone (PT) which can be implemented as a combination of quadrupoles: 


$$
\mathbf{M}_{\mathrm{PT}}\left(\phi_{x}, \phi_{y}\right)=\left(\begin{array}{cc}
\mathbf{M}_{x x}\left(\phi_{x}\right) & \mathbf{0} \\
\mathbf{0} & \mathbf{M}_{y y}\left(\phi_{y}\right)
\end{array}\right)
$$

it is equivalent to $T_{\text {decoupled }}\left(\phi_{x}, \phi_{y}\right)$ used in [5].

Rotation in $x-y$ plane can be performed using a fictitious element beam rotator $(\mathrm{BR})$ which can be implemented as a combination of quadrupoles, skew-quadrupoles and/or solenoids:

$$
\begin{aligned}
\mathbf{M}_{\mathrm{BR}}\left(\phi_{c}\right)= & \left(\begin{array}{cc}
\mathbf{M}_{x 0}(0) & \mathbf{0} \\
\mathbf{0} & \mathbf{M}_{y 0}(0)
\end{array}\right) \mathbf{R}\left(\phi_{c}\right)\left(\begin{array}{ccc}
\mathbf{M}_{0 x}(0) & \mathbf{0} \\
\mathbf{0} & \mathbf{M}_{0 y}(0)
\end{array}\right) \\
= & \left(\begin{array}{cccc}
\cos \phi_{c} & 0 & c_{x y} & 0 \\
0 & \cos \phi_{c} & \frac{\alpha_{y}-\alpha_{x}}{\sqrt{\beta_{x} \beta_{y}}} \sin \phi_{c} & c_{y x} \\
-c_{y x} & 0 & \cos \phi_{c} & 0 \\
\frac{\alpha_{y}-\alpha_{x}}{\sqrt{\beta_{x} \beta_{y}}} \sin \phi_{c} & -c_{x y} & 0 & \cos \phi_{c}
\end{array}\right),
\end{aligned}
$$

where $c_{x y}=\sqrt{\beta_{x} / \beta_{y}} \sin \phi_{c}, c_{y x}=\sqrt{\beta_{y} / \beta_{x}} \sin \phi_{c}, \mathbf{R}(\phi)$ is a matrix of the beam rotation around longitudinal axis:

$$
\mathbf{R}(\phi)=\left(\begin{array}{cccc}
\cos \phi & 0 & \sin \phi & 0 \\
0 & \cos \phi & 0 & \sin \phi \\
-\sin \phi & 0 & \cos \phi & 0 \\
0 & -\sin \phi & 0 & \cos \phi
\end{array}\right) .
$$

It transforms $\beta_{x, y}, \alpha_{x, y}$ to some equal values $\beta_{0}, \alpha_{0}$, performs beam rotation and transforms back. Note that the final expression is independent on the intermediate values $\beta_{0}$ and $\alpha_{0}$. An important restriction is that beam rotator does not change particle distribution in the beam only in case of equal emittances, $\varepsilon_{x}=\varepsilon_{y}$, which is approximately the case for MESA and S-DALINAC. Transformation $T_{\text {coupled }}\left(\phi_{1}, \phi_{2}\right)$ used in [5] is a combination of a phase trombone and $90^{\circ}$-rotation in $x-y$ plane.

To use the fictitious elements described above one should insert their transport matrices into certain positions in the lattice. Within an arc their positions are not important, for better visualization the same points near the centers of recirculation arcs were chosen for both of them. Results of 1D and 2D optimization over $\phi_{c}$ and $\left(\phi_{x}, \phi_{y}\right)$ will be presented in the next section. Global optimization involving all the inserted elements is possible, but to save computation time, it was performed separately for each arc.

\section{SIMULATION RESULTS}

\section{A. Cross-check between tracking and stability analysis}

An example of the cross-check procedure between the two techniques for one of dipole mode pairs is presented below. In Fig. 7 the central part of the complex current plot is shown (threshold current value is marked with a red point). Figures 8 and 9 show time dependencies of the

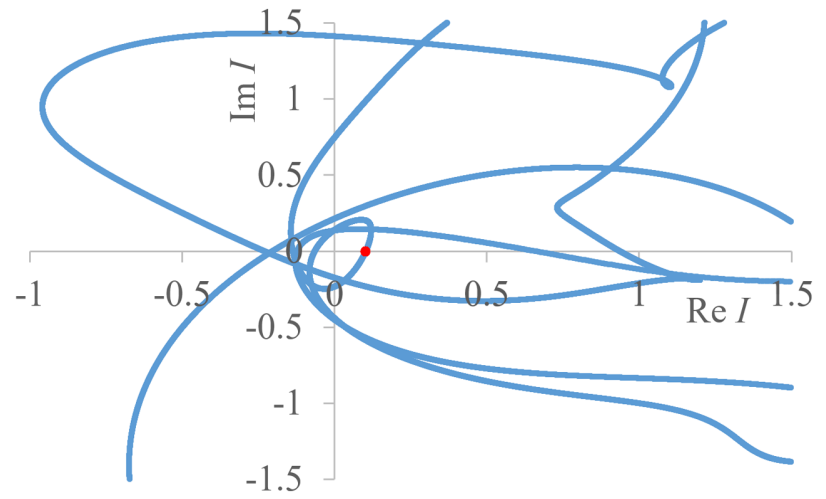

FIG. 7. Complex current plot for one of the mode configurations in MESA (only one pair of dipole modes is active). The threshold current value is marked with a red point.

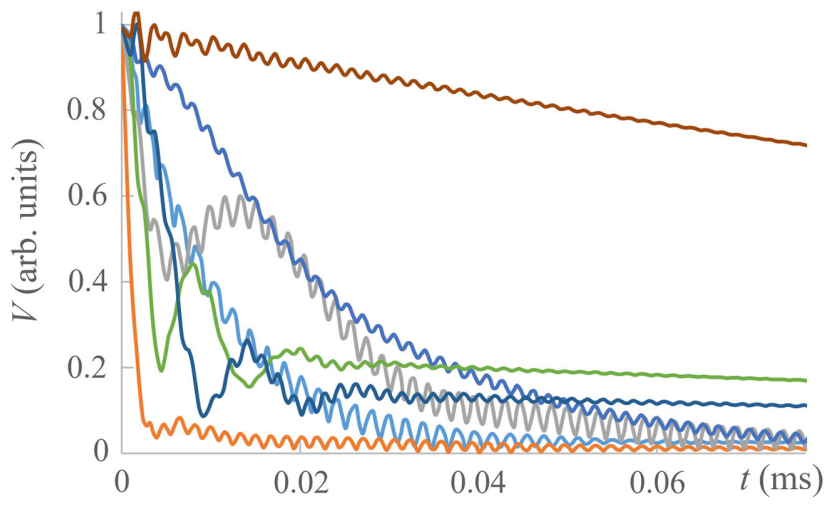

FIG. 8. Dipole mode voltages time dependence for a current $10 \%$ below the threshold. Mode configuration is the same as in Fig. 7.

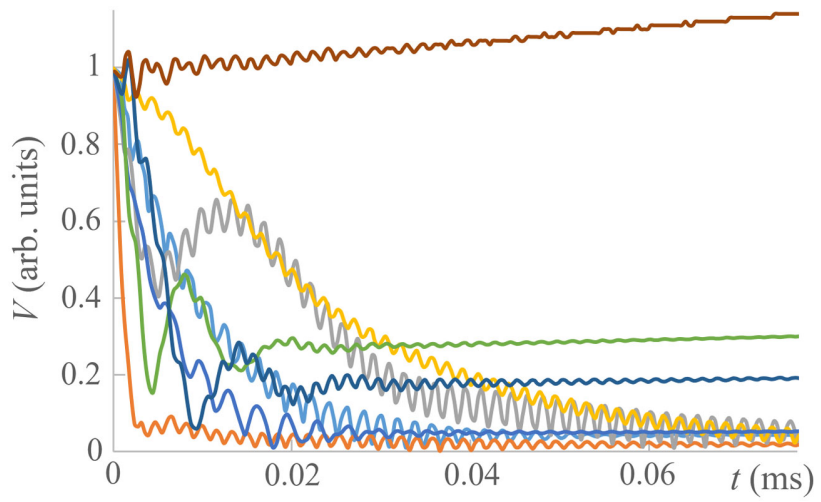

FIG. 9. Dipole mode voltages time dependence for a current $10 \%$ above the threshold. Mode configuration is the same as in Fig. 7.

absolute values of mode voltages for beam current values $10 \%$ below and above the threshold correspondingly. It can be seen that after a relatively short transition process mode 
voltages begin to constantly fall (below threshold) or rise (above threshold).

A similar procedure with automated and visual check has been performed for several tens of different dipole and quadrupole mode configurations in MESA and S-DALINAC. Agreement is always much better than $\pm 10 \%$. This can be regarded as a proof of applicability of the approximated stability analysis technique developed in this work, at least to these facilities. Detailed comparison to the exact technique [4] is also planned.

\section{B. Search for the most dangerous modes}

To save simulation time, modes with the strongest impact were selected for further study. For this purpose 100 random mode configurations of MESA and S-DALINAC were generated. Then BBU threshold current was calculated separately for each monopole mode and $n_{\lambda} \geq 1$ mode pair in each configuration and minimum over all configurations was found. Results are shown in Figs. 10 and 11.

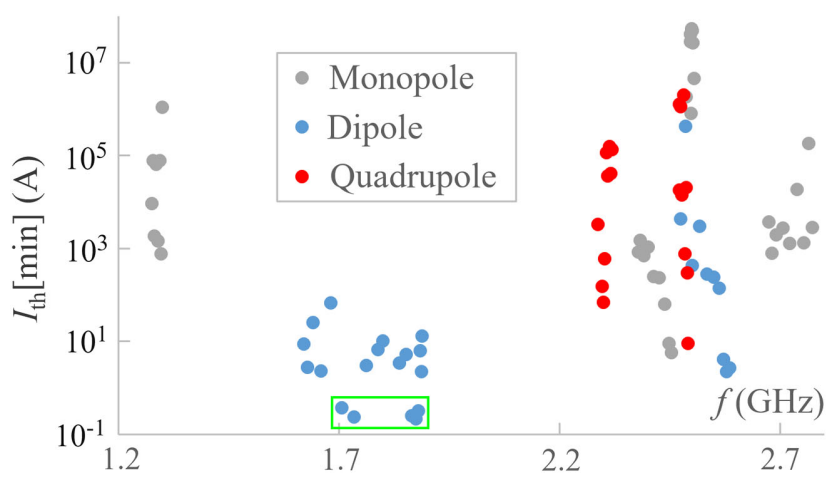

FIG. 10. Minimal threshold current for different modes in MESA. Five of the most dangerous dipole mode pairs are enclosed in a green box.

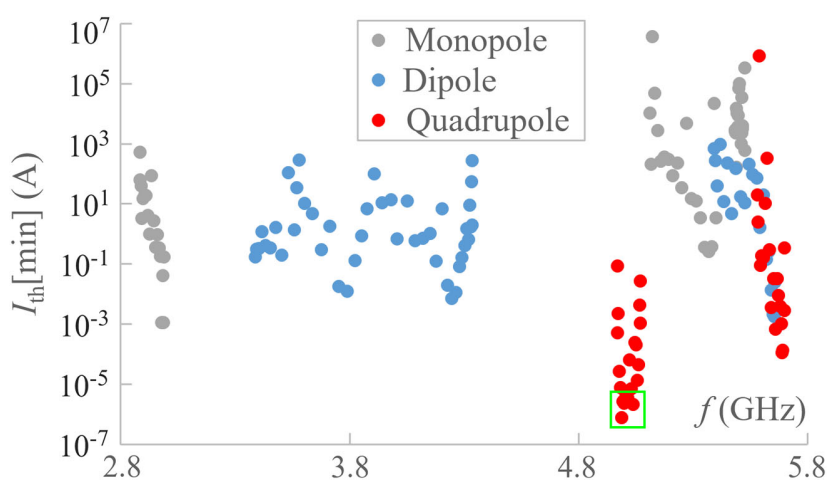

FIG. 11. Minimal threshold current for different modes in S-DALINAC. Four of the most dangerous quadrupole mode pairs are enclosed in a green box.
As it was predicted above, the most dangerous are the modes from the first two dipole passbands in MESA and from the first quadrupole passband in S-DALINAC. Threshold current values for the lowest two dipole passbands in MESA are in accordance with the results presented in [32].

\section{Optimization results}

Combinations of thin phase trombones (PT) and beam rotators (BR) were inserted near the centers of T1, T2, T3 arcs of MESA and F, S arcs of S-DALINAC. During each optimization run only one of these elements was controlled while others were disabled. Examples of 2D PT-scan and 1D BR-scan results for 5 the most dangerous dipole mode pairs in MESA are presented in Figs. 12 and 13 correspondingly. Figure of merit here is the maximal threshold current value which can be achieved with a particular BBU mitigation technique. However the minimal achievable value is also important to characterize the effectiveness of the technique. Also spread of the minimal and maximal

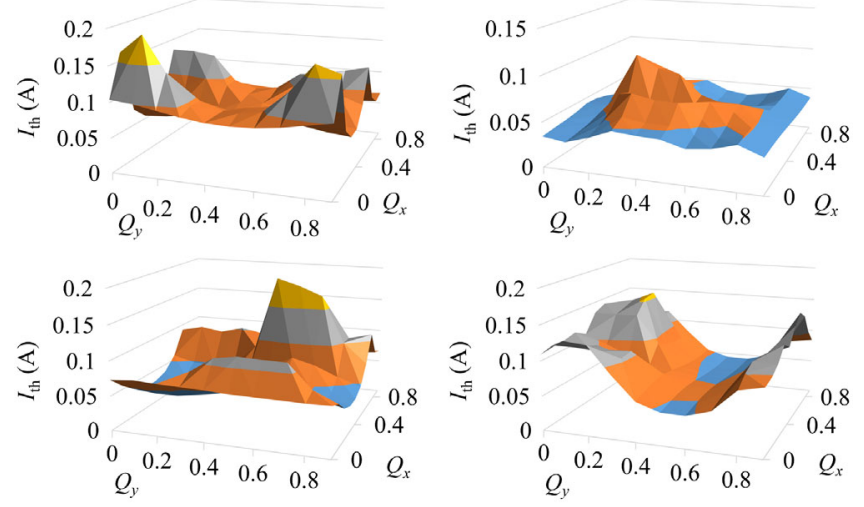

FIG. 12. Examples of 2D phase trombone scans for different configurations of $\operatorname{MESA}\left(Q_{x, y}=\phi_{x, y} / 2 \pi\right)$.
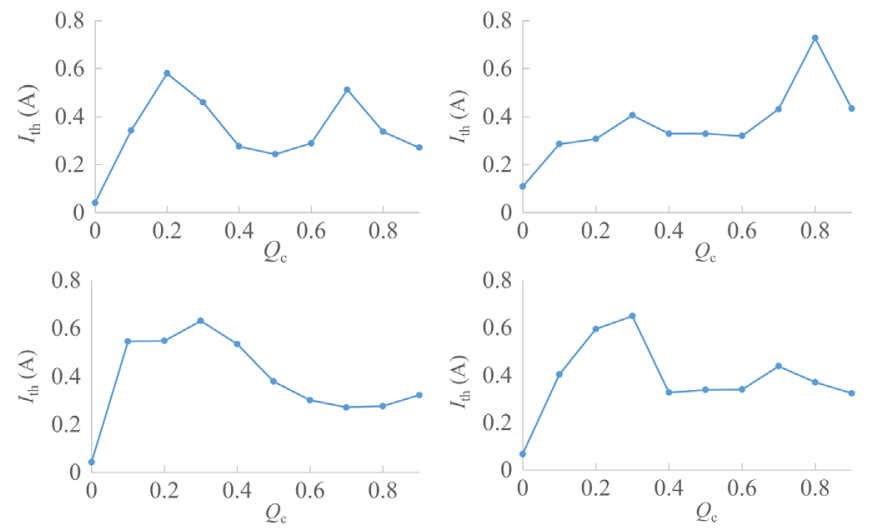

FIG. 13. Examples of 1D beam rotator scans for different configurations of MESA $\left(Q_{c}=\phi_{c} / 2 \pi\right)$. 
TABLE IV. Effectiveness of different BBU mitigation techniques in MESA.

\begin{tabular}{lccc}
\hline \hline & Technique & $I_{\mathrm{th}_{\text {min }}}(\mathrm{A})$ & $I_{\mathrm{th}_{\max }}(\mathrm{A})$ \\
\hline 1 & PT in T1 arc & $0.047 \pm 0.022$ & $1.15 \pm 0.65$ \\
2 & PT in T2 arc & $0.029 \pm 0.012$ & $1.06 \pm 0.51$ \\
3 & PT in T3 arc & $0.029 \pm 0.018$ & $0.65 \pm 0.22$ \\
4 & BR in T1 arc & $0.072 \pm 0.037$ & $3.00 \pm 1.44$ \\
5 & BR in T2 arc & $0.072 \pm 0.037$ & $2.31 \pm 1.29$ \\
6 & BR in T3 arc & $0.072 \pm 0.037$ & $1.55 \pm 0.71$ \\
\hline \hline
\end{tabular}

TABLE V. Effectiveness of different BBU mitigation techniques in S-DALINAC.

\begin{tabular}{lccc}
\hline \hline & Technique & $I_{\mathrm{th}_{\min }}(\mu \mathrm{A})$ & $I_{\mathrm{th}_{\max }}(\mu \mathrm{A})$ \\
\hline 1 & PT in F arc & $0.0428 \pm 0.0404$ & $537 \pm 534$ \\
2 & PT in S arc & $0.415 \pm 0.406$ & $467 \pm 392$ \\
4 & BR in F arc & $1.34 \pm 1.27$ & $105 \pm 94.6$ \\
5 & BR in S arc & $0.00301 \pm 0.00282$ & $95.7 \pm 95.3$ \\
\hline \hline
\end{tabular}

values over different randomized configurations helps to understand if the positive effect of this particular technique is guaranteed or not. Combination of these four values can be regarded as a universal criterion of the optimization effectiveness.

Final results calculated for different BBU mitigation techniques in 10 randomized configurations of MESA and S-DALINAC are presented in Tables IV and V as well as in Figs. 14 and 15. It can be seen that for each technique there is a clear gap between minimal and maximal values, including spread, therefore, each of them should be effective.

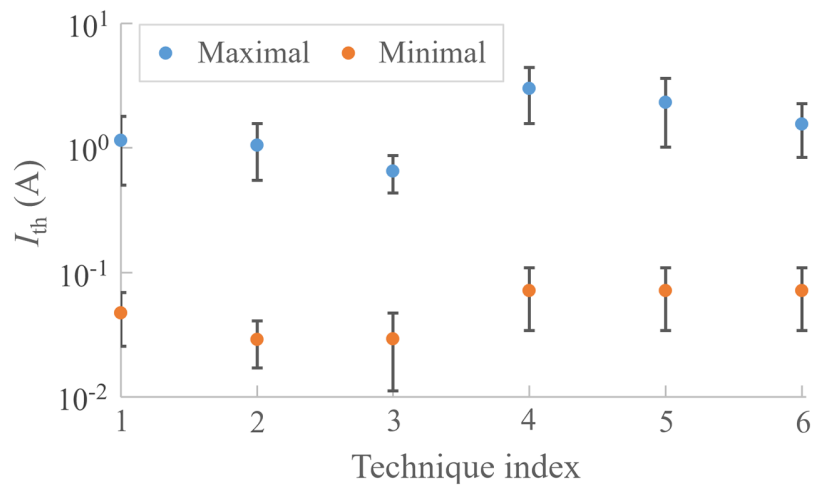

FIG. 14. Minimal and maximal threshold current values achievable with different BBU mitigation techniques for 10 randomized configurations of MESA.

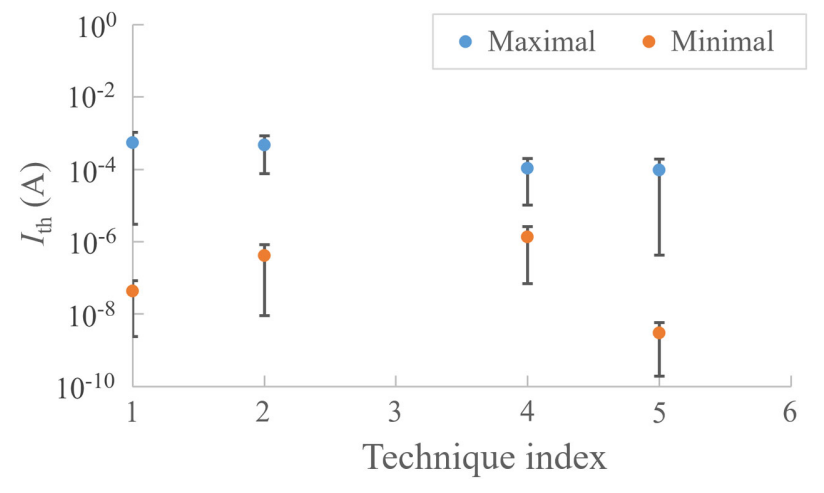

FIG. 15. Minimal and maximal threshold current values achievable with different BBU mitigation techniques for 10 randomized configurations of S-DALINAC.

\section{CONCLUSION}

In this study an attempt was taken to consider the BBU phenomenon in a more general way while keeping formulas as simple as possible. In particular, the BBU caused by monopole, dipole and quadrupole electromagmetic modes in rf cavities were studied together using similar frameworks. Therefore, tracking results can be easily verified using the stability analysis technique initially developed only for dipole modes.

The general approach to BBU mitigation techniques related to linear lattice modifications proposed in [5] was extended and illustrated with the examples for two different recirculating machines, MESA and S-DALINAC. The universal criterion proposed in [5] which can be used for effectiveness assessment of different techniques was also extended.

There are three main outcomes from the study with respect to S-DALINAC and MESA: 1. The approximated stability analysis technique can be used in the case of multiple recirculations to achieve better performance. Detailed comparison to the results obtained with the exact technique [4] is planned as the next step of the present research. 2. Quadrupole modes can be more dangerous in terms of BBU in some particular machines (e.g., S-DALINAC). Therefore, one should always take them into account in BBU studies. 3. Tools for changing and measurement of betatron phase advances as well as betatron coupling should be always included into the design of new recirculating machines; they can be also introduced into existing machines. It allows to increase the BBU threshold current without usage of expensive dedicated components.

The experimental validation of the described model is also planned at S-DALINAC. It will include the BBU threshold current measurements with different quadrupole settings (betatron tune scan). Observations of the recirculated beam behavior close to the BBU threshold are also planned to determine the type of the BBU-causing modes (dipole or quadrupole). As it was already mentioned, 
S-DALINAC cavities have no HOM couplers, which means that the voltages and actual parameter values of the parasitic modes cannot be measured. Therefore, the variety of possible BBU experiments at S-DALINAC is significantly limited. The diagnostics possibilities at the future MESA ERL will be much larger.

A simple model of a damped oscillator with feedback is proposed in Appendix B as a universal example illustrating the BBU phenomenon in general. This model cannot be used for the BBU threshold current calculations in a particular machine but it clearly demonstrates the essence of the instability.

\section{ACKNOWLEDGMENTS}

This work has been supported by DFG through Research Training Group GRK 2128 "AccelencE-Accelerator
Science and Technology for Energy-Recovery Linacs". The authors are grateful to Norbert Pietralla, Ruben Grewe, Felix Schließmann, Wolfgang Ackermann, Wolfgang Müller, Herbert De Gersem, Uwe Niedermayer (TU Darmstadt), Kurt Aulenbacher, Florian Hug, Daniel Simon (JGU Mainz) for a lot of fruitful discussions, manuscript reviewing and providing the data necessary for the simulations.

\section{APPENDIX A: COEFFICIENTS FOR BBU STABILITY ANALYSIS WITH QUADRUPOLE MODES}

Coefficients in expression Eq. (10) have the following form:

$$
\begin{array}{rl}
a_{\mu \lambda}^{l}= & 2\left[\left(T_{31} T_{32}-T_{11} T_{12}\right) \Sigma_{11}+\left(T_{32}^{2}-T_{12}^{2}\right) \Sigma_{12}+\left(T_{11} T_{14}-T_{31} T_{34}+T_{32} T_{33}-T_{12} T_{13}\right) \Sigma_{13}\right. \\
& \left.+\left(T_{32} T_{34}-T_{12} T_{14}\right) \Sigma_{14}+\left(T_{12} T_{14}-T_{32} T_{34}\right) \Sigma_{23}+\left(T_{13} T_{14}-T_{33} T_{34}\right) \Sigma_{33}+\left(T_{14}^{2}-T_{34}\right) \Sigma_{34}\right], \\
b_{\mu \lambda}^{l}= & 2\left[\left(T_{11} T_{14}-T_{31} T_{34}\right) \Sigma_{11}+\left(T_{12} T_{14}-T_{32} T_{34}\right) \Sigma_{12}+\left(T_{11} T_{12}-T_{31} T_{32}+T_{13} T_{14}-T_{33} T_{34}\right) \Sigma_{13}\right. \\
+ & \left.\left(T_{14}^{2}-T_{34}^{2}\right) \Sigma_{14}+\left(T_{12}^{2}-T_{32}^{2}\right) \Sigma_{23}+\left(T_{12} T_{13}-T_{32} T_{33}\right) \Sigma_{33}+\left(T_{12} T_{14}-T_{32} T_{34}\right) \Sigma_{34}\right], \\
c_{\mu \lambda}^{l}= & 2\left[-\left(T_{11} T_{32}+T_{12} T_{31}\right) \Sigma_{11}-2 T_{12} T_{32} \Sigma_{12}+\left(T_{14} T_{31}-T_{12} T_{33}+T_{11} T_{34}-T_{13} T_{32}\right) \Sigma_{13}\right. \\
& \left.-\left(T_{12} T_{34}+T_{14} T_{32}\right) \Sigma_{14}+\left(T_{14} T_{32}+T_{12} T_{34}\right) \Sigma_{23}+\left(T_{13} T_{34}+T_{14} T_{33}\right) \Sigma_{33}+2 T_{14} T_{34} \Sigma_{34}\right], \\
& \\
d_{\mu \lambda}^{l}=2 & 2\left(T_{11} T_{34}+T_{14} T_{31}\right) \Sigma_{11}+\left(T_{14} T_{32}+T_{12} T_{34}\right) \Sigma_{12}+\left(T_{14} T_{33}+T_{11} T_{32}+T_{12} T_{31}+T_{13} T_{34}\right) \Sigma_{13} \\
+ & \left.2 T_{14} T_{34} \Sigma_{14}+2 T_{12} T_{32} \Sigma_{23}+\left(T_{13} T_{32}+T_{12} T_{33}\right) \Sigma_{33}+\left(T_{14} T_{32}+T_{12} T_{34}\right) \Sigma_{34}\right],
\end{array}
$$

where indices ${ }_{\mu \lambda}^{l}$ are omitted in all $\left(T_{\mu \lambda}^{l}\right)_{i j}$ and $\left(\Sigma_{\mu \lambda}^{l}\right)_{i j}$ for brevity.

\section{APPENDIX B: ILLUSTRATION OF BBU PHENOMENON}

After considering BBU mechanisms and development of the techniques for BBU simulations and stability analysis which can be used in real machines, one may want to study the simplest possible system showing similar behavior. Obviously, in terms of recirculating machines this should be a lattice with one recirculation arc and single cavity, where only one dipole mode can be excited (dipole mode should be chosen to keep the problem as linear as possible). For further simplification bunched beam can be replaced with a coasting beam continuously injected into the machine. Needless to say, such beam cannot be accelerated or decelerated using conventional means, but now it is not needed. Moreover, acceleration or deceleration is a result of beam interaction with the fundamental mode which is also not necessary for the simplest example, therefore, cavity excitation can be just switched off. Then the voltage $u(t)$ of the only mode existing in the cavity can be described with a differential equation of a damped oscillator with a feedback (driven by itself via delay loop):

$$
\ddot{u}(t)+b \dot{u}(t)+\omega^{2} u(t)=k u(t-\tau),
$$

where $\omega$ is the mode frequency, $b=\omega /(2 Q)$ is damping coefficient, $k$ is feedback strength, $\tau$ is feedback loop delay time. For Eq. (B1) to describe a linear or nonlinear oscillator, its right-hand side should be a linear or nonlinear function of $u(t)$, correspondingly. But presence of the linear term with a time delay provides some unique properties while partly keeping linear behavior. In particular, the equation has a 
harmonic solution $u(t)=C e^{z t}$, which leads to the following dispersion relation:

$$
z^{2}+b z+\omega^{2}=k e^{-z \tau}
$$

but depending on $k$ the solution may be either stable or unstable. To find $k=k_{\text {th }}$ corresponding to a separatrix between stable and unstable solutions, one should consider purely imaginary values $z=i y$, where $y \in \mathbb{R}$, then

$$
\begin{aligned}
\omega^{2}-y^{2} & =k_{\mathrm{th}} \cos \tau y, \\
b y & =-k_{\mathrm{th}} \sin \tau y .
\end{aligned}
$$

Without loss of generality, these equations can be multiplied by $\tau^{2}$ in order to switch to dimensionless parameters $\varphi=\omega \tau, \psi=y \tau, B=b \tau, K=k_{\mathrm{th}} \tau^{2}$ :

$$
\begin{aligned}
\varphi^{2}-\psi^{2} & =K \cos \psi, \\
B \psi & =-K \sin \psi,
\end{aligned}
$$

which can be rewritten as

$$
\begin{gathered}
B \cot \psi=\psi-\frac{\varphi^{2}}{\psi}, \\
K^{2}=\left(\varphi^{2}-\psi^{2}\right)^{2}+(B \psi)^{2} .
\end{gathered}
$$

Equation (B2) has multiple solutions which can be found numerically but only one of them providing minimum to $K$ in Eq. (B3) should be chosen. Therefore, only $\psi$ values around $\psi_{*}=\sqrt{\varphi^{2}-B^{2} / 2}$ should be checked in Eq. (B2).

Figures 16 and 17 show $K(\varphi)$ dependency at two different values of $B$. The graph is confined between lines $K=B \varphi$ and $K=\sqrt{2 B} \varphi$ which can be also proven theoretically. Moreover, it can be approximated as follows:

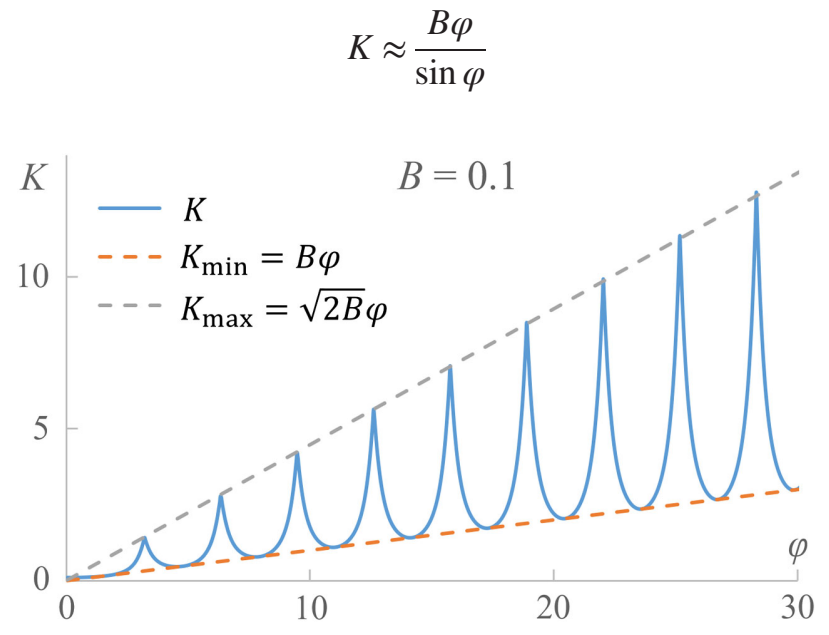

FIG. 16. $K(\varphi)$ dependency at $B=0.1$.

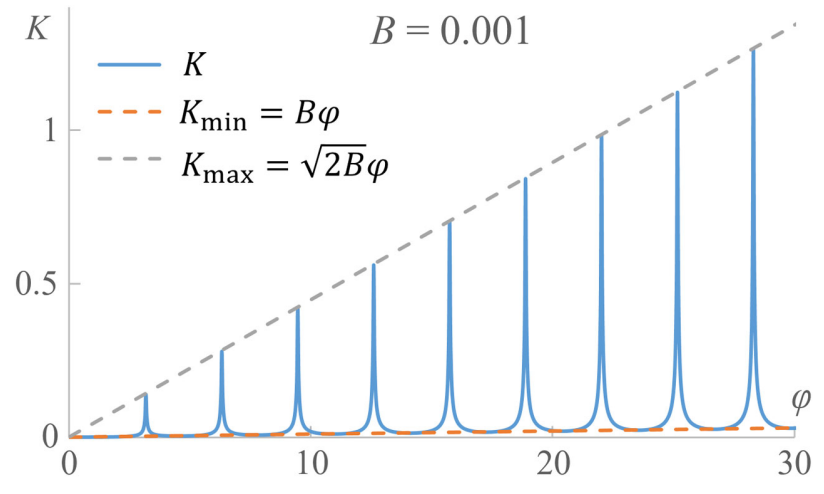

FIG. 17. $K(\varphi)$ dependency at $B=0.001$.

in the region $K \leq \sqrt{2 B} \varphi$, which resembles the approximate formula for the BBU threshold current from [4].

The example under study can be further extended to illustrate applicability of the eigenvalue approach proposed in [3]. Now consider a set of $N$ oscillators with frequencies $\omega_{i}$ and damping factors $b_{i}$ pairwise connected via feedback loops, multiple loops are possible for each pair. $I k_{i j l}$ and $\tau_{i j l}$ are strengths and delay times of $n_{i j}$ feedback loops between $i$ th and $j$ th oscillator, then

$\ddot{u}_{i}(t)+b_{i} \dot{u}_{i}(t)+\omega_{i}^{2} u_{i}(t)=I \sum_{j=1}^{N}\left[\sum_{l=1}^{n_{i j}} k_{i j l} u\left(t-\tau_{i j l}\right)\right]$.

These oscillators correspond to all possible modes which can be excited in the cavities of the machine; multiple feedback loops between the same oscillators correspond to multiple recirculations; common factor $I$ in feedback strengths corresponds to the beam current. System of equations (B4) has a set of harmonic solutions

$$
u_{i}(t)=C_{i} e^{z t},
$$

leading to a system of dispersion relations

$$
C_{i}\left(z^{2}+b_{i} z+\omega_{i}^{2}\right)=I \sum_{j=1}^{N} C_{j}\left(\sum_{l=1}^{n_{i j}} k_{i j l} e^{-z \tau_{i j l}}\right) .
$$

which is a linear equation system on $C_{i}$. It can be solved only when $1 / I$ is an eigenvalue of a matrix with the following elements:

$$
M_{i j}(z)=\frac{\sum_{l=1}^{n_{i j}} k_{i j l} e^{-z \tau_{i j l}}}{z^{2}+b_{i} z+\omega_{i}^{2}} .
$$

Therefore, coupled system of linear damped oscillators driven by themselves via multiple delay loops shows similar behavior to a system without delays and can be studied using conventional techniques. The main difference caused by the 
delays is a nontrivial shape of the stability region in parameter space. Certain form of this stability region cannot be predicted using this simplified model, numerical analysis is necessary for real machines. However, one should be aware of the capabilities and limitations of such analysis which were illustrated using the model.

One can find more detailed information on systems with feedback and time delay from the point of view of control theory in $[33,34]$. Certain similarities can be found between complex current plot and Nyquist plot which is widely used for stability analysis in control theory. However, here emphasis is not placed on them to keep the example as simple as possible.

[1] L. Merminga, Energy recovery linacs, in Synchrotron Light Sources and Free-electron Lasers: Accelerator Physics, Instrumentation and Science Applications, edited by E. J. Jaeschke, S. Khan, J. R. Schneider, and J. B. Hastings (Springer International Publishing, Cham, 2016) pp. 419-457.

[2] R. H. Helm and G. A. Loew, Beam breakup, in Linear Accelerators, edited by P. Lapostolle et al. (North-Holland, Amsterdam, 1970) pp. 173-221.

[3] G. Krafft, J. Bisognano, and S. Laubach, Calculating beam breakup in superconducting linear accelerators (1990), https://www.osti.gov/biblio/882282.

[4] G. H. Hoffstaetter and I. V. Bazarov, Beam-breakup instability theory for energy recovery linacs, Phys. Rev. ST Accel. Beams 7, 054401 (2004).

[5] W. Lou and G. H. Hoffstaetter, Beam breakup current limit in multiturn energy recovery linear accelerators, Phys. Rev. Accel. Beams 22, 112801 (2019).

[6] J. J. Bisognano and M. L. Fripp, Requirements for longitudinal HOM damping in superconducting recirculating linacs, (1989), https://accelconf.web.cern.ch/188/papers/ we3-32.pdf.

[7] B. C. Yunn, A beam breakup instability in a recirculating linac caused by a quadrupole mode, in Proceedings of the 2003 Particle Accelerator Conference (IEEE, New York, 2003).

[8] N. Pietralla, The institute of nuclear physics at the TU Darmstadt, Nucl. Phys. News 28, 4 (2018).

[9] F. Hug, K. Aulenbacher, S. Friederich, P. Heil, R. Heine, R. Kempf, C. Matejcek, and D. Simon, Status of the MESA ERL project, in 63rd ICFA Advanced Beam Dynamics Workshop on Energy Recovery Linacs (JACoW Publishing, Berlin, 2020).

[10] M. Abo-Bakr et al., The Berlin Energy Recovery Linac Project BERLinPro - status, plans and future opportunities, in 63rd ICFA Advanced Beam Dynamics Workshop on Energy Recovery Linacs (JACoW Publishing, Berlin, 2020).

[11] A. Bartnik, N. Banerjee, D. Burke, J. Crittenden, K. Deitrick, J. Dobbins et al., CBETA: First Multipass Superconducting Linear Accelerator with Energy Recovery, Phys. Rev. Lett. 125, 044803 (2020).
[12] W. Kaabi et al., PERLE: A high power energy recovery facility, in 10th International Particle Accelerator Conference (JACoW Publishing, Melbourne, 2019).

[13] N. Vinokurov et al., Novosibirsk ERL facility, in 59th ICFA Advanced Beam Dynamics Workshop on Energy Recovery Linacs (JACoW Publishing, Geneva, 2018).

[14] M. Arnold, C. Eschelbach, R. Grewe, F. Hug, T. Kürzeder, M. Lösler, J. Pforr, and N. Pietralla, Construction and status of the thrice recirculating S-DALINAC, in 8th International Particle Accelerator Conference (JACoW Publishing, 2017).

[15] M. Arnold, C. Burandt, C. Eschelbach, T. Kürzeder, M. Lösler, J. Pforr, and N. Pietralla, ERL mode of S-DALINAC: Design and status, in 59th ICFA Advanced Beam Dynamics Workshop on Energy Recovery Linacs (JACoW Publishing, Geneva, 2018).

[16] M. Arnold, J. Birkhan, J. Pforr, N. Pietralla, F. Schließmann, M. Steinhorst, and F. Hug, First operation of the superconducting Darmstadt linear electron accelerator as an energy recovery linac, Phys. Rev. Accel. Beams 23, 020101 (2020).

[17] B. Aune et al., The superconducting TESLA cavities, Phys. Rev. ST Accel. Beams 3, 092001 (2000).

[18] F. Hug, M. Arnold, L. Jürgensen, T. Kürzeder, N. Pietralla, and M. Schilling, Beam dynamics calculations and magnet design for future measurements of transverse beam breakup at the S-DALINAC, in 27th International Linear Accelerator Conference (JACoW Publishing, Geneva, 2014).

[19] M. Arnold, C. Burandt, C. Eschelbach, R. Grewe, F. Hug, M. Lösler, J. Pforr, N. Pietralla, and M. Steinhorst, First ERL operation of S-DALINAC and commissioning of a path length adjustment system, in 9th International Particle Accelerator Conference (JACoW Publishing, Vancouver, 2018).

[20] E. Jensen, RF cavity design, in CERN Accelerator School: Advanced Accelerator Physics Course (2014) arXiv: 1601.05230 .

[21] CST MICROWAVE STUDIO. Workflow \& solver overview, CST GmbH - Computer Simulation Technology (1998-2007), https://www.rose-hulman.edu/class/ee/ HTML/ECE340/PDFs/MWS_Tutorials.pdf.

[22] M. Borland, Features and applications of the program ELEGANT, in 4th International Particle Accelerator Conference (JACoW Publishing, Shanghai, 2013).

[23] W. Herr and F. Schmidt, A MAD-X primer, in CERN Accelerator School and DESY Zeuthen: Accelerator Physics (2004).

[24] I. Bazarov, bi - Beam Instability Code, Cornell University, Ithaca, NY, 14853 (Cornell University, Ithaca, 2003), https:// www.classe.cornell.edu/ ib38/bbucode/doc/bbudoc.pdf.

[25] T. Stengler, K. Aulenbacher, F. Hug, T. Kürzeder, and D. Simon, Cryomodule fabrication and modification for high current operation at the Mainz Energy Recovering Superconducting Accelerator MESA, in 18th International Conference on RF Superconductivity (JACoW Publishing, Lanzhou, 2018).

[26] T. Stengler, K. Aulenbacher, F. Hug, and S. Thomas, SRF testing for Mainz Energy Recovering Superconducting Accelerator MESA, in 19th International Conference on 
RF Superconductivity (SRF 2019) (JACoW Publishing, Dresden, 2019).

[27] L. Xiao, C. Adolphsen, V. Akcelik, A. Kabel, K. Ko, L. Lee, $\mathrm{Z}$. $\mathrm{Li}$, and $\mathrm{C}$. $\mathrm{Ng}$, Modeling imperfection effects on dipole modes in TESLA cavity, in Contributed to Particle Accelerator Conference (PAC 2007), 6/25/2007-6/29/2007, Albuquerque (2007).

[28] V. N. Litvinenko, Chromaticity of the lattice and beam stability in energy recovery linacs, Phys. Rev. ST Accel. Beams 15, 074401 (2012); 18, 039901(E) (2015).

[29] S. Setiniyaz, R. Apsimon, and P. H. Williams Id, Filling pattern dependence of regenerative beam breakup instability in energy recovery linacs, arXiv:2104.13418.

[30] J. Rosenzweig and L. Serafini, Transverse particle motion in radiofrequency linear accelerators, Phys. Rev. E 49, 1599 (1994).
[31] R. E. Rand and T. I. Smith, Beam optical control of beam breakup in a recirculating electron accelerator, in Particle Accelerators (Gordon and Breach, Science Publishers, 1980), Vol. 11, pp. 1-3.

[32] C. Stoll and F. Hug, Beam breakup simulations for the Mainz Energy Recovering Superconducting Accelerator MESA, J. Phys. Conf. Ser. 1350, 012111 (2019).

[33] H. Klingbeil, U. Laier, and D. Lens, Theoretical Foundations of Synchrotron and Storage Ring RF Systems, Particle Acceleration and Detection (Springer, Cham, 2015).

[34] H. Unbehauen and F. Ley, Hütte - Das Ingenieurwissen (Springer Berlin Heidelberg, Berlin, Heidelberg, 2008). 